\title{
THE LICK-CARNEGIE EXOPLANET SURVEY: GLIESE 687 b-A NEPTUNE-MASS PLANET ORBITING A NEARBY RED DWARF
}

\author{
Jennifer Burt ${ }^{1}$, Steven S. Vogt ${ }^{1}$, R. Paul Butler ${ }^{2}$, Russell Hanson $^{1}$, Stefano Meschiari $^{3}$, \\ Eugenio J. Rivera ${ }^{1}$, Gregory W. Henry ${ }^{4}$, ANd Gregory Laughlin ${ }^{1}$ \\ ${ }^{1}$ UCO/Lick Observatory, Department of Astronomy and Astrophysics, University of California at Santa Cruz, Santa Cruz, CA 95064, USA \\ ${ }^{2}$ Department of Terrestrial Magnetism, Carnegie Institute of Washington, Washington, DC 20015, USA \\ ${ }^{3}$ McDonald Observatory, University of Texas at Austin, Austin, TX 78752, USA \\ ${ }^{4}$ Center of Excellence in Information Systems, Tennessee State University, Nashville, TN 37209, USA \\ Received 2014 February 24; accepted 2014 May 8; published 2014 June 20
}

\begin{abstract}
Precision radial velocities from the Automated Planet Finder (APF) and Keck/HIRES reveal an $M \sin (i)=$ $18 \pm 2 M_{\oplus}$ planet orbiting the nearby M3V star GJ 687. This planet has an orbital period $P=38.14$ days and a low orbital eccentricity. Our Strömgren $b$ and $y$ photometry of the host star suggests a stellar rotation signature with a period of $P=60$ days. The star is somewhat chromospherically active, with a spot filling factor estimated to be several percent. The rotationally induced 60 day signal, however, is well separated from the period of the radial velocity variations, instilling confidence in the interpretation of a Keplerian origin for the observed velocity variations. Although GJ $687 \mathrm{~b}$ produces relatively little specific interest in connection with its individual properties, a compelling case can be argued that it is worthy of remark as an eminently typical, yet at a distance of $4.52 \mathrm{pc}$, a very nearby representative of the galactic planetary census. The detection of GJ $687 \mathrm{~b}$ indicates that the APF telescope is well suited to the discovery of low-mass planets orbiting low-mass stars in the as yet relatively un-surveyed region of the sky near the north celestial pole.
\end{abstract}

Key words: planets and satellites: detection - planets and satellites: gaseous planets - stars: individual (GL687)

Online-only material: color figures

\section{INTRODUCTION}

The Copernican principle implies that the Earth, and, by extension, the solar system, do not hold a central or specifically favored position. This viewpoint is related to the so-called mediocrity principle (Kukla 2010), which notes that an item drawn at random is more likely to come from a heavily populated category than one which is sparsely populated.

These principles, however, have not had particularly apparent success when applied in the context of extrasolar planets. Mayor et al. (2009) used their high precision Doppler survey data to deduce that of order 50\% (or more) of the chromospherically quiet main-sequence dwarf stars in the solar neighborhood are accompanied by a planet (and in many cases, by multiple planets) with $M \sin (i) \lesssim 30 M_{\oplus}$, and orbital periods of $P<$ 100 days. Taken strictly at face value, this result implies that our own solar system, which contains nothing interior to Mercury's $P=88$ day orbit, did not participate in the galaxy's dominant mode of planet formation. Yet the eight planets of the solar system have provided, and continue to provide, the de facto template for most discussions of planet formation.

Indeed, where extrasolar planets are concerned, $\mathrm{M}$ dwarfs and mediocrity appear to be effectively synonymous. Recent observational results suggest that low-mass planets orbiting lowmass primaries are by no means rare. Numerous examples of planets with $M_{\mathrm{p}}<30 M_{\oplus}$ and $\mathrm{M}$ dwarf primaries have been reported by the Doppler surveys (e.g., Butler et al. 2004; Mayor et al. 2009, and many others), and the Kepler Mission has indicated that small planets are frequent companions to lowmass stars. For example, Dressing \& Charbonneau (2013) report that among dwarf stars with $T_{\text {eff }}<4000 \mathrm{~K}$, the occurrence rate of $0.5 R_{\oplus}<R_{\mathrm{p}}<4 R_{\oplus}$ planets with $P<50$ days is $N=0.9_{-0.03}^{+0.04}$ planets per star. Improved statistics, however, are required for a definitive statement that is couched in planetary masses as well as in planetary radii. Figure 1 shows the current distribution of reported planets and planetary candidates orbiting primaries with $M_{\star}<0.6 M_{\odot}$, which we adopt as the functional border between "M-type" stars and "K-type stars."

The census of low-mass planets orbiting low-mass primaries can be accessed using a variety of techniques. For objects near the bottom of the main sequence, it appears that transit photometry from either ground (Charbonneau 2010) or space (Triaud et al. 2013) offer the best prospects for planetary discovery and characterization. For early to mid M-type dwarfs, there is a large enough population of sufficiently bright primaries that precise Doppler detection (see, e.g., Rivera et al. 2010) can play a lead role. For the past decade, we have had a sample of $\sim 160$ nearby, photometrically quiet M-type stars under precision radial velocity (RV) surveillance with the Keck telescope and its HIRES spectrometer. In recent months, this survey has been supplemented by data from the Automated Planet Finder (APF) telescope (Vogt et al. 2014b). Here, we present $16.6 \mathrm{yr}$ of Doppler velocity measurements for the nearby M3 dwarf GJ 687 (including 122 velocity measurements from Keck, 20 velocity measurements from the APF, and 5 velocity measurements made with the Hobby-Eberly Telescope (HET)) and we report the detection of the exoplanet that they imply. We use this discovery of what is a highly archetypal representative of a planet in the Milky Way-in terms of its parent star, its planetary mass, and its orbital period - to motivate a larger discussion of the frequency of occurrence, physical properties, and detectability of low-mass planets orbiting M-type stars.

The plan for this paper is as follows. In Section 2, we describe the physical and spectroscopic properties of the red dwarf host star Gliese 687. In Section 3, we describe our RV observations of this star. In Section 4 we describe our Keplerian model for these 


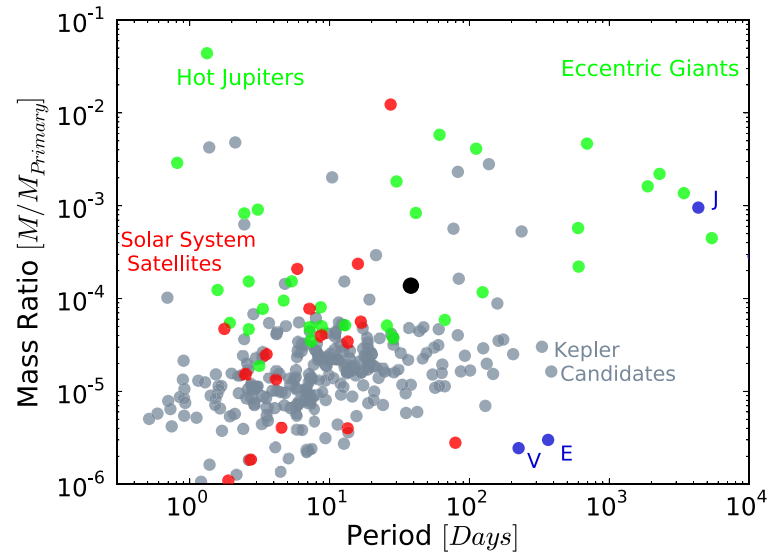

Figure 1. Population diagram for currently known extrasolar planets orbiting stars with reported masses $M_{\text {star }}<0.6 M_{\odot}$. Green circles: planets securely detected by the radial velocity method (either with or without photometric transits). Red circles: the regular satellites of the Jovian planets in the solar system. Gray circles: Kepler candidates and objects of interest. Radii for these candidate planets, as reported in (Batalha et al. 2013), have been converted to masses assuming $M / M_{\oplus}=\left(R / R_{\oplus}\right)^{2.06}$ (Lissauer et al. 2011), which is obtained by fitting the masses and radii of the solar system planets bounded in mass by Venus and Saturn, which may be a rather naive transformation given the startling range of observed radii for planets with masses between Earth and Uranus. Venus, Earth, and Jupiter are indicated on the diagram for comparison purposes. Data are from www.exoplanets.org, accessed 2014 December 1.

(A color version of this figure is available in the online journal.)

observations, along with an analysis that assesses our confidence in the detection. In Section 5, we describe our photometric time series data for the star, which aids in the validation of the planet by ruling out spot-modulated interpretations of the Doppler variations. In Section 6, we discuss the ongoing refinement of the planet-metallicity correlation for low-mass primaries, in Section 7, we discuss the overall statistics that have emerged from more than $15 \mathrm{yr}$ of precision Doppler observations of M dwarf stars with the Keck Telescope, and in Section 8 we conclude with an overview that evaluates the important future role of the APF telescope in precision velocimetry of nearby, low-mass stars.

\section{GJ 687 STELLAR PARAMETERS}

Gliese 687 (LHS 450, BD+68 946) lies at a distance, $d=4.5 \mathrm{pc}$, is the 39 th nearest known stellar system, and is the closest star north of $+60^{\circ}$ declination. Figure 2 indicates GJ 687's position in the color-magnitude diagram (CMD) for stars in the Lick-Carnegie Survey's database of Keck observations. Due to its proximity and its brightness ( $V=9.15$, $K_{\mathrm{s}}=4.548$ ), Gliese 687 has been heavily studied, and in particular, the CHARA Array has recently been used to obtain direct interferometric angular diameter measurements for the star. Boyajian et al. (2012) find $R_{\star} / R_{\odot}=0.4183 \pm 0.0070$, and derive $L_{\star} / L_{\odot}=0.02128 \pm 0.00023, T_{\text {eff }}=3413 \mathrm{~K}$, and use the mass-radius relation of Henry \& McCarthy (1993) to obtain $M_{\star} / M_{\odot}=0.413 \pm 0.041$. As illustrated in Figure 3, Gliese 687's mean Mt. Wilson S value (calculated per Wright et al. 2004) and the dispersion of its S-index measurements from our spectra indicate that it has a moderate degree of chromospheric activity. This conclusion is in concordance with our long-term photometric monitoring program, which also indicates that the star is somewhat active.

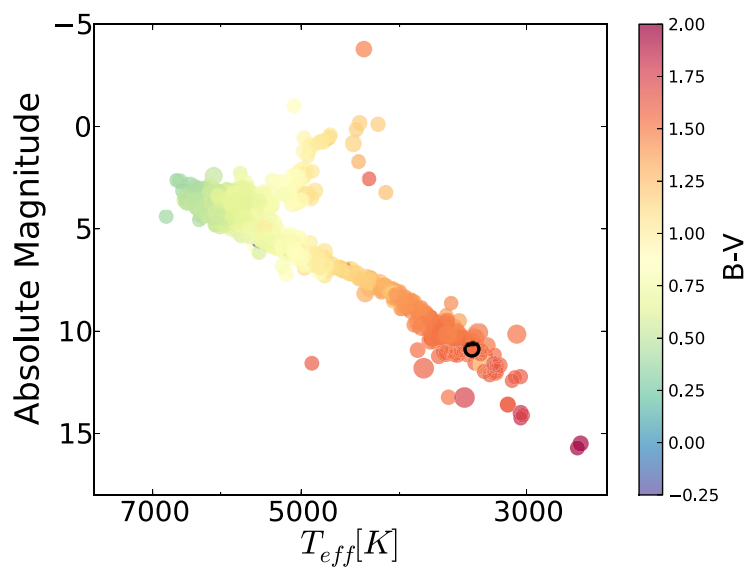

Figure 2. H-R diagram with GJ 687's position indicated as a small open circle. Absolute magnitudes, $M$, are estimated from $V$ band apparent magnitudes and Hipparcos distances using $M=V+5 \log _{10}(d / 10 \mathrm{pc})$. All 956 stars in our catalog of radial velocity measurements (for which more than 20 Doppler measurements exist) are shown, color-coded by their $B-V$ values, with point areas sized according to the number of observations taken.

(A color version of this figure is available in the online journal.)

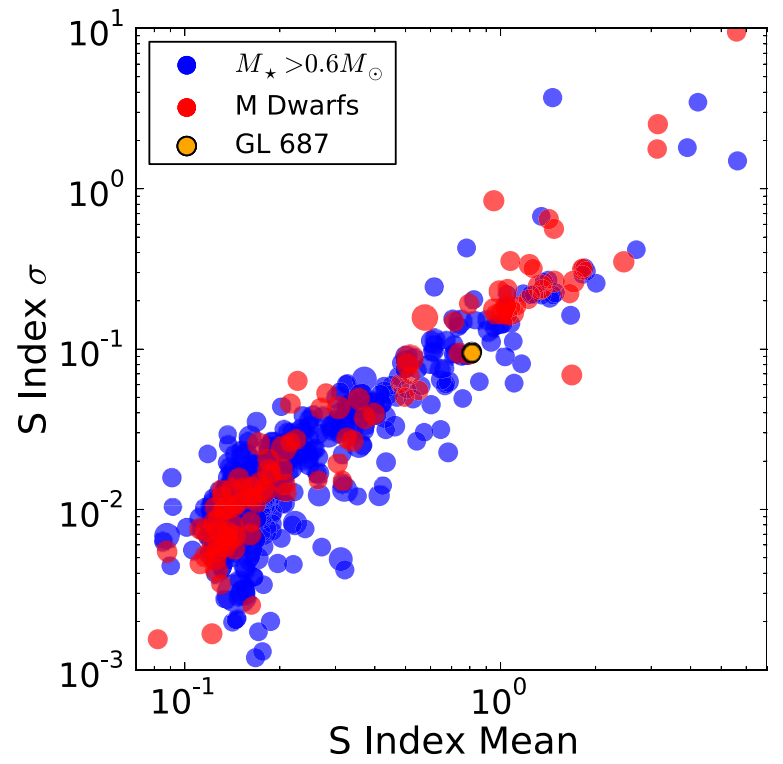

Figure 3. Average value of the $S$-index against the standard deviation of the $\mathrm{S}$-index for all the stars in the Lick-Carnegie database. Stars with $M_{\star}<0.6 M_{\odot}$ are colored red. GJ 687 is shown as an orange circle in the midst of this population, showing that it is a somewhat active star. The areas subtended by the individual points are, in all cases, proportional to the number of Doppler velocity observations that we have collected of the star (with systems above an upper bound of 250 observations receiving the same point size).

(A color version of this figure is available in the online journal.)

\section{RADIAL VELOCITY OBSERVATIONS}

Doppler shifts from both the Keck (122 observations) and APF (20 observations) platforms were measured, in each case, by placing an iodine absorption cell just ahead of the spectrometer slit in the converging beam of stellar light from the telescope (Butler et al. 1996). The forest of iodine lines superimposed on the stellar spectra generates a wavelength calibration and enables measurement of each spectrometer's point-spread function (PSF). The RVs from Keck were obtained by operating HIRES at a spectral resolving power $R \sim 70,000$ over the wavelength range of 3700-8000 $\AA$, though only the region 5000-6200 


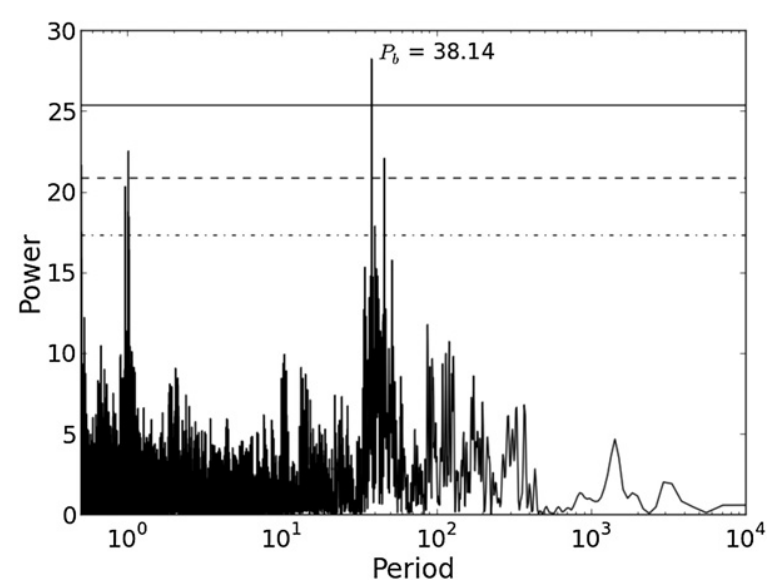

Figure 4. Lomb-Scargle periodograms for combined radial velocity measurements of GJ 687 from the HET, Keck, and APF telescopes. The horizontal lines from top to bottom represent false alarm probabilities of $0.01 \%, 0.1 \%$, and $1.0 \%$ respectively.

containing a significant density of iodine lines was used in the present Doppler analysis (Vogt et al. 1994). The APF measurements were obtained over a similar spectral range, but at a higher spectral resolving power, $R \sim 108,000$. For each spectrum that was obtained, the region containing the iodine lines was divided into $\sim 700$ chunks, each of $\sim 2 \AA$ width. Each chunk produces an independent measure of the wavelength, PSF, and Doppler shift. The final measured velocity is the weighted mean of the velocities of the individual chunks. All RVs have been corrected to the solar system barycenter, but are not tied to any absolute velocity system. As such, they are "relative" velocities, with a zero point that can float as a free parameter within an overall system model.

The internal uncertainties quoted for all the RV measurements in this paper reflect only one term in the overall error budget, and result from a host of errors that stem from the characterization and determination of the PSF, detector imperfections, optical aberrations, consequences of undersampling the iodine lines, and other effects. Two additional major sources of error are photon statistics and stellar "jitter." The latter varies widely from star to star, and can be mitigated to some degree by selecting magnetically inactive older stars and by time-averaging over the star's unresolved low-degree surface $p$-modes. All observations in this paper have been binned on $2 \mathrm{hr}$ timescales. In addition to the RVs that we have obtained at Keck and APF, we also use five Doppler measurements obtained by Endl et al. (2003) at the HET located at McDonald Observatory. These RV observations are presented in the Appendix.

\section{THE BEST FIT SOLUTION}

The combined RV data sets show a root-mean-square (rms) scatter of $7.58 \mathrm{~m} \mathrm{~s}^{-1}$ about the mean velocity. This scatter is measured after we have applied best-fit telescope offsets of $0.64 \mathrm{~m} \mathrm{~s}^{-1}$ for Keck, $-1.71 \mathrm{~m} \mathrm{~s}^{-1}$ for APF, and $1.27 \mathrm{~m} \mathrm{~s}^{-1}$ for HET.

A Lomb-Scargle periodogram of the 149 velocity measurements of GJ 687 is shown in Figure 4. False alarm probabilities (FAPs) are calculated with the bootstrap method, as described in Efron (1979), iterating 100,000 times for a minimum probability of $P_{\text {false }}<1 e-5$ as easily met by the tallest $P_{\mathrm{b}}=$ 38.14 day peak in Figure 4. This signal in the data is modeled as a $M_{\mathrm{b}} \sin (i)=0.06 M_{J}$ planet with an orbital eccentricity, $e_{b}=0.04$.

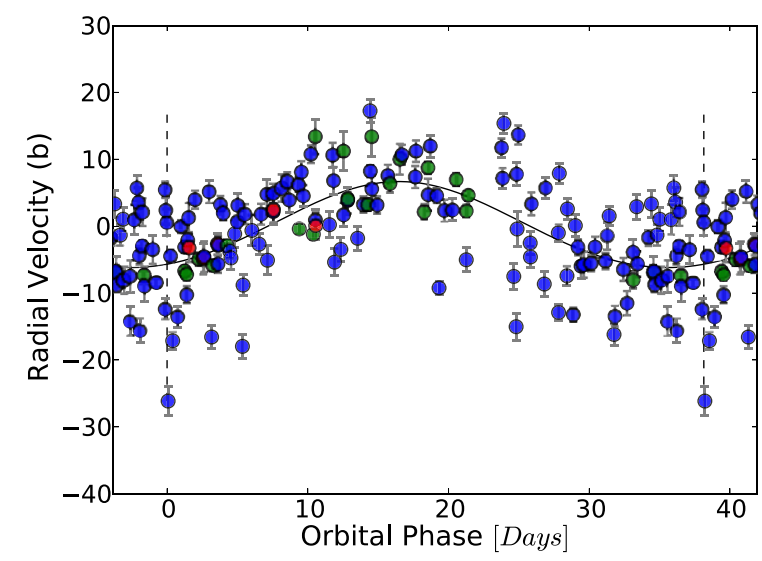

Figure 5. Phased radial velocity model for planet b, folded at the $P=38.14$ day orbital period. The blue points correspond to Keck data points, green points are APF data, and the red points are HET data. The vertical dashed lines demarcate the extent of unique data.

(A color version of this figure is available in the online journal.)

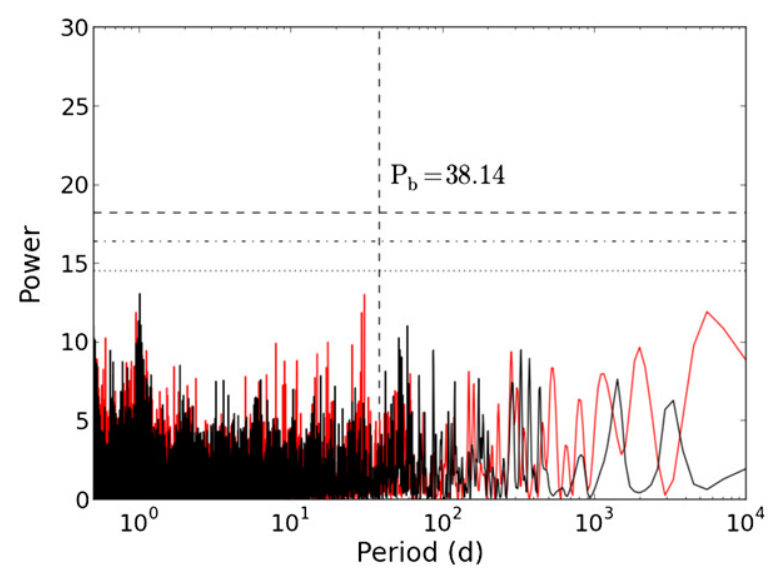

Figure 6. Lomb-Scargle periodogram of the radial velocity residuals to the fit given in Table 2 plotted in black, and the Lomb-Scargle periodogram of the Mt. Wilson S-index values plotted behind in red.

(A color version of this figure is available in the online journal.)

Using Levenberg-Marquardt optimization, we obtained a best-fit Keplerian model for the system. This fit, which assumes $i=90^{\circ}$ and $\Omega=0^{\circ}$ for the planet, is listed in Table 2 . The phased RV curve for the planet in Table 2 is shown in Figure 5. A power spectrum of the residuals to our one-planet fit is shown in Figure 6 and indicates no significant periodicities. Also shown in this figure is a periodogram of our Mt. Wilson $\mathrm{S}$-index measurements from the spectra, which are a proxy for the degree of spot activity on the star at a given moment. None of the peaks in the periodogram of S-index values coincide with the peak that we suspect to be a planet.

The reduced chi-squared statistic for our fit is $\chi_{\text {red }}^{2}=18.55$ and results in a fit with a combined rms of $6.16 \mathrm{~m} \mathrm{~s}^{-1}$ and estimated excess variance of $\sigma_{\mathrm{jitter}}=5.93 \mathrm{~m} \mathrm{~s}^{-1}$ (the estimate of the jitter that is required to bring the reduced chi-squared statistic of the fit down to unity). This value accounts for variance in both the stellar signal and from the telescope itself, though for a moderately active star such as GJ 687 a stellar jitter of order $6.0 \mathrm{~m} \mathrm{~s}^{-1}$ is reasonable, and could account for the majority of the observed variance.

In order to compute parameter uncertainties for our orbital fit, we implement a Markov Chain Monte Carlo algorithm (Ford 


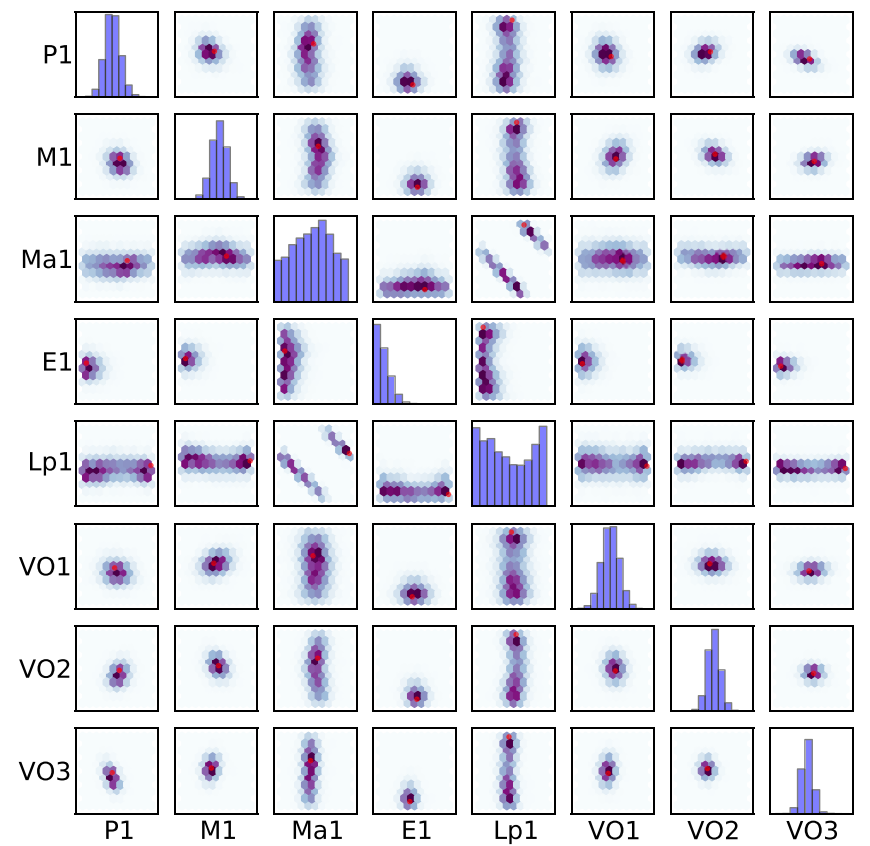

Figure 7. Smooth scatter plots of parameter error correlations for our Markov chain. In each case, the best-fit model is indicated with a small red dot, and the density of models within the converged portion of the chain is shown as a blue-toned probability distribution function. The diagonal line of entries shows the marginalized distribution for each parameter of the one-planet model.

(A color version of this figure is available in the online journal.)

2005; Ford \& Gaudi 2006; Balan \& Lahav 2009; Meschiari et al. 2009; Gregory 2011). The MCMC algorithm returns a chain of state vectors, $\mathbf{k}_{i}$ (a set of coupled orbital elements, e.g., period, mass, etc. and the three velocity offset parameters). The goal of the Markov Chain calculation is to generate an equilibrium distribution proportional to $\exp \left[\chi^{2}(\mathbf{k})\right]$. We adopt non-informative priors on all parameters (and uniform in the $\log$ for masses and periods). The resulting error correlations are shown in Figure 7, and a set of 100 states drawn randomly from the converged chain are shown in the orbital diagram in Figure 8.

The error correlation diagram indicates that all parameters are well determined, save the usual degeneracies between mean anomaly and $\omega$ for the low-eccentricity orbit. The distribution of the residuals relative to the best-fit model shows no evident pathologies. Indeed, a quantile-quantile plot (shown in Figure 9) indicates that the distribution of residuals is well described by a normal distribution. We note that the smaller scatter of points obtained with the APF telescope could be a consequence of the fact that they were all taken within a $\Delta t=140 \mathrm{~d}$ period, and thus sample only one segment of the stellar activity cycle.

A potentially significant challenge to correctly identifying the orbital period of a proposed exoplanet arises from the discrete and uneven sampling inherent in RV surveys. The spacing of observations leads to increased noise and the presence of aliases within the star's periodogram which can be mistaken for a true orbital signature. For a real signal occurring at a frequency $f_{\text {planet }}$ we expect alias signatures at $f=f_{\text {planet }} \pm n f_{\text {sampling }}$ where $\mathrm{n}$ is an integer. In order to aid confirmation that the periodic signal we observe is actually a planetary signature, we must be able to calculate where aliases due to our observing cadence will occur, and then verify that they are not the source of the signal. The aliases are determined using a spectral window function as

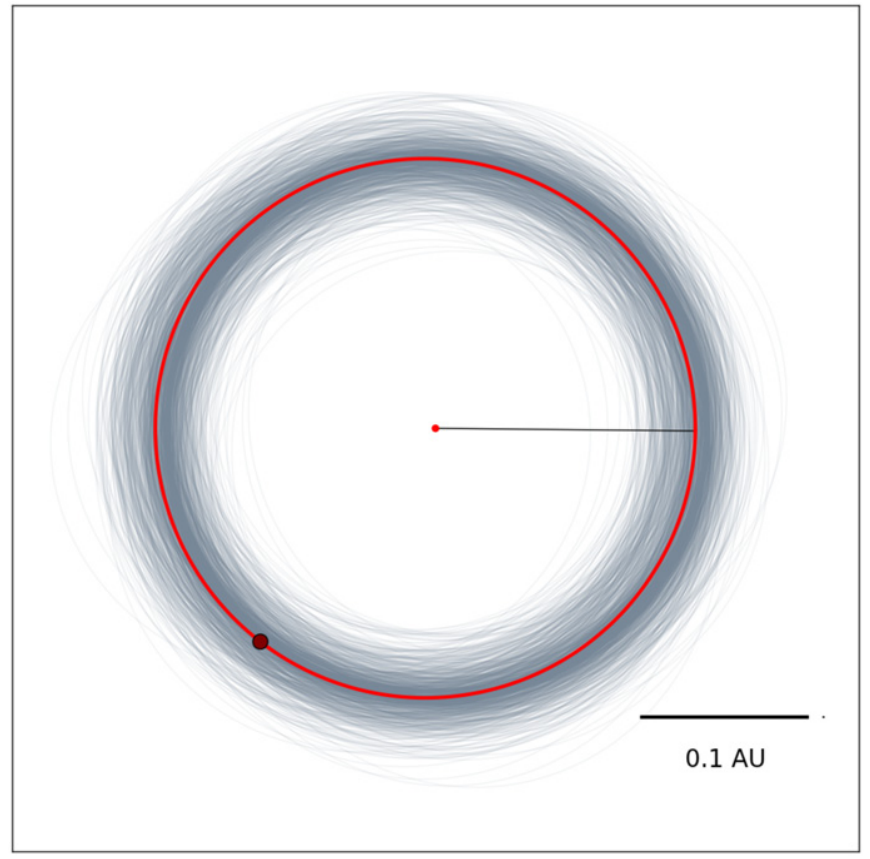

Figure 8. Orbit of the proposed planetary companion to GJ 687. The larger red point corresponds to the location of the planet at the initial observation epoch, HJD 2450603.97. The line from the origin corresponds to the planet's periastron. For the geometry plotted, transits, should they occur, would happen when the planet traverses the positive $y$-axis. The light lines are 100 orbits of the planet drawn from the converged segment of the Markov Chain. The red dot in the center of the diagram corresponds to the size of the star when drawn to scale. The small black dot next to the distance scale bar indicates the size of the planet when drawn to scale, and assuming it has $R_{\mathrm{P}}=R_{\mathrm{Nep}}$.

(A color version of this figure is available in the online journal.)

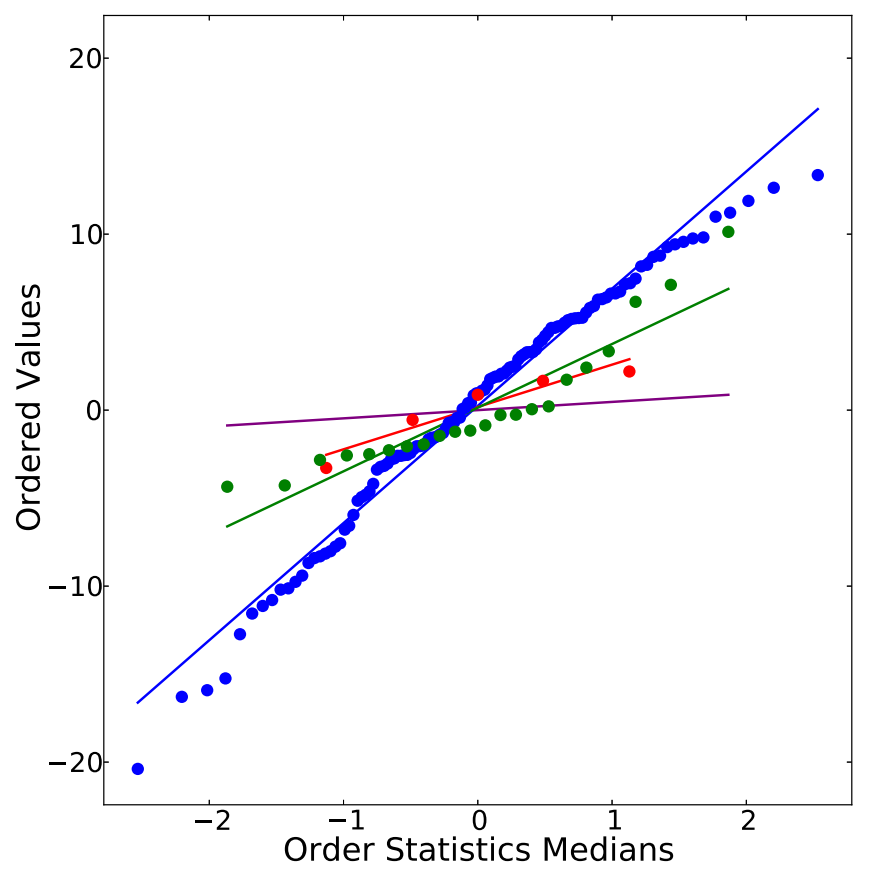

Figure 9. Quantile-quantile plot for the velocity residuals to the one-planet model fit. Adherence of the points to the lines indicate the degree to which the radial velocities from the two telescopes conform to a normal distribution. APF points are shown in green, Keck points are shown in blue, and HET points are shown in red.

(A color version of this figure is available in the online journal.) 


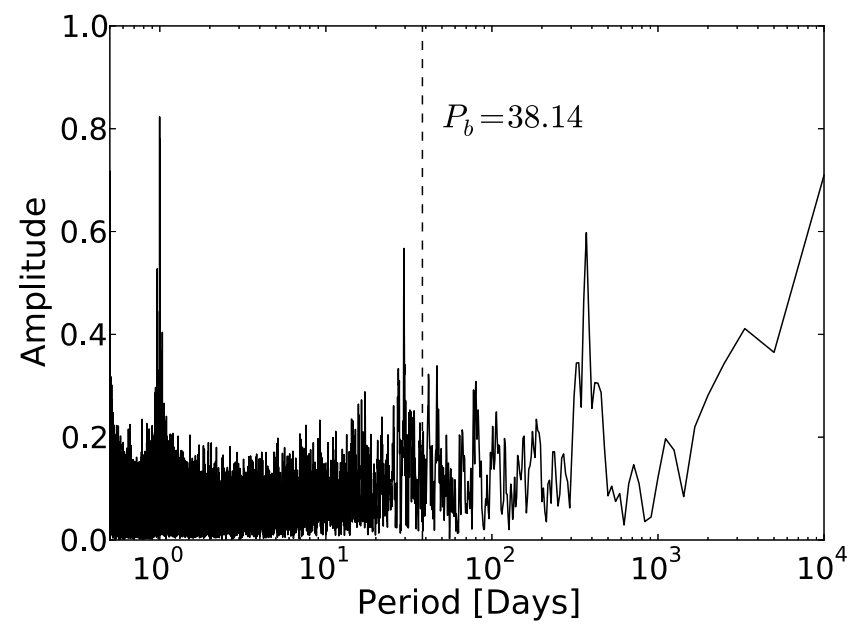

Figure 10. Window function calculated from all radial velocity observations of GL 687. While several peaks exist due to aliasing effects from our data's time stamps, none of them coincide with the locations necessary to create a peak in the periodogram at our best fit period of $P=38.14$ days.

defined by Roberts et al. (1987):

$$
W(v)=\frac{1}{N} \sum_{r=0}^{N} \exp ^{-2 \pi i v t_{r}}
$$

where $N$ is the total number of observations and $t$ is the date on which they were taken. Plotting this function will result in peaks that are due solely to the sampling cadence of the data. Because our observations are constrained by when the star is visible in the night sky, and because Keck Telescope time is allocated to Doppler surveys primarily when the Moon is up, we expect aliases at periods of 1 solar day, 1 sidereal day, 1 synodic month and 1 sidereal year. Examining the window function in Figure 10 we do see peaks resulting at these periods, but careful analysis of the periodogram for our RV observations shows no evidence of strong signals occurring at the locations necessary for our $P=38.14$ day signal to be a potential alias instead of a true Keplerian signature.

With an apparent $K_{\mathrm{s}}$-band magnitude of 4.54 , Gliese 687 is brighter (in the near infrared) than all known hosts of transiting extrasolar planets other than 55 Cancri. As a consequence, transits by Gliese 687's planetary companion (which has an equilibrium temperature, $T_{\mathrm{eq}} \sim 260 \mathrm{~K}$ ), were they to occur, would be of substantial scientific value. In particular, transmission spectroscopy with James Webb Space Telescope (JWST) would give insights into what is likely a dynamic and chemically rich planetary atmosphere. The a priori geometric transit probability for Gliese $687 \mathrm{~b}$, however, is a scant $P_{\mathrm{tr}}=1.2 \%$, and as we describe below, there is no evidence that transits occur. With $M \sin (i)=19 M_{\oplus}$, the currently observed mass-radius range for exoplanets indicates that the planetary radius, $R_{\mathrm{p}}$ could credibly range from $R_{\mathrm{p}} \sim 0.2 R_{\text {Jup }}$ to $R_{\mathrm{p}} \sim 0.6 R_{\text {Jup }}$, implying potential transit depths in the $d=0.2 \%$ to $d=2 \%$ range.

\section{PHOTOMETRIC OBSERVATIONS}

During the 2009-2013 observing seasons, we acquired a total of 866 photometric observations of GJ 687 on 519 nights with the Tennessee State University (TSU) T12 $0.80 \mathrm{~m}$ automatic photoelectric telescope (APT) at Fairborn Observatory in
Arizona. The T12 APT is one of several TSU automatic telescopes operated at Fairborn (Henry 1999; Eaton et al. 2003). It is equipped with a two-channel precision photometer that employs a dichroic filter and standard Strömgren $b$ and $y$ filters to separate the two passbands and two EMI 9124QB bi-alkali photomultiplier tubes to measure the $b$ and $y$ count rates simultaneously. We observed GJ 687, designated our program star (P), differentially with respect to three neighboring comparison stars: C1 (HD 156295, $V=5.54, B-V=0.22$, F0 IV), C2 (HD 160198, $V=7.65, B-V=0.46$, F2 V), and C3 (HD 161538, $V=7.01, B-V=0.44, \mathrm{~F} 2 \mathrm{~V})$. A detailed description of the observing sequence and the data reduction and calibration procedures are given in Henry (1999).

We computed all pairwise differential magnitudes $P-C 1$, $P-C 2, P-C 3, C 3-C 2, C 3-C 1$, and $C 2-C 1$ in both the $b$ and $y$ passbands, corrected them for atmospheric extinction, and transformed them to the standard Strömgren photometric system. Observations with internal standard deviations greater than $0.01 \mathrm{mag}$ were discarded to remove data taken in non-photometric conditions. Intercomparison of the six sets of differential magnitudes demonstrated that HD 156295 (C1) is a low-amplitude variable while both HD 160198 (C2) and HD 161538 (C3) are constant to the expected measurement precision. To improve our precision, we combined the separate differential $b$ and $y$ observations into a single $(b+y) / 2$ "passband." We also computed the differential magnitudes of GJ 687 with respect to the mean brightness of the two good comparison stars: $P-(C 2+C 3) / 2$. The standard deviation of the $C 3-C 2$ comparison star differential magnitudes is $0.0020 \mathrm{mag}$, which we take to be the precision of a single measurement.

A total of 606 nightly measurements in the five observing seasons survived the cloud-filtering process. These data are plotted as $P-(C 2+C 3) / 2$ differential magnitudes in the top panel of Figure 11. The five individual observing seasons are plotted in the remaining panels. The standard deviations for the yearly light curves are given in each panel. These range from 0.0049 to $0.0092 \mathrm{mag}$, compared to the measurement precision of $0.0020 \mathrm{mag}$. Gaps of 10-12 weeks in the yearly light curves for 2009 through 2012 are due to southern Arizona's July-September rainy season when good photometry is not possible.

Low-amplitude variability is seen in GJ 687 during each observing season, resembling light curves typical of modestly active stars with spot filling factors of a few percent (see, e.g., Henry et al. 1995). The 2010 light curve has the largest amplitude variability $(\sim 0.03 \mathrm{mag})$ and reveals cyclic variation with a time scale of $\sim 60$ days. The other light curves have lower amplitudes and include cyclic variations of $\sim 60$ and also $\sim 30$ days. These year-to-year and cycle-to-cycle variations are also typical of modestly active stars. We interpret the 60 day variability as the signature of the star's rotation period and the 30 day variability as a sign of spot activity on opposite hemispheres of the star. This slow rotation rate is in agreement with the work done by Jenkins et al. (2009) which reports an upper rotational velocity of $2.8 \mathrm{~km} \mathrm{~s}^{-1}$ for GL 687 .

Frequency spectra of the complete 2009-2013 data set and of the 2010 data alone are shown in the top and bottom panels of Figure 12 respectively. The rotational modulation signal is seen most clearly in the 2010 data, which matches up with the most coherent light curve in Figure 11. Therefore, we take the $58.48 \pm 1.0$ days signal as our best measurement of the star's rotation period. Inspection of the 2010 photometric segment of Figure 11 clearly shows the overall 60 day modulation 

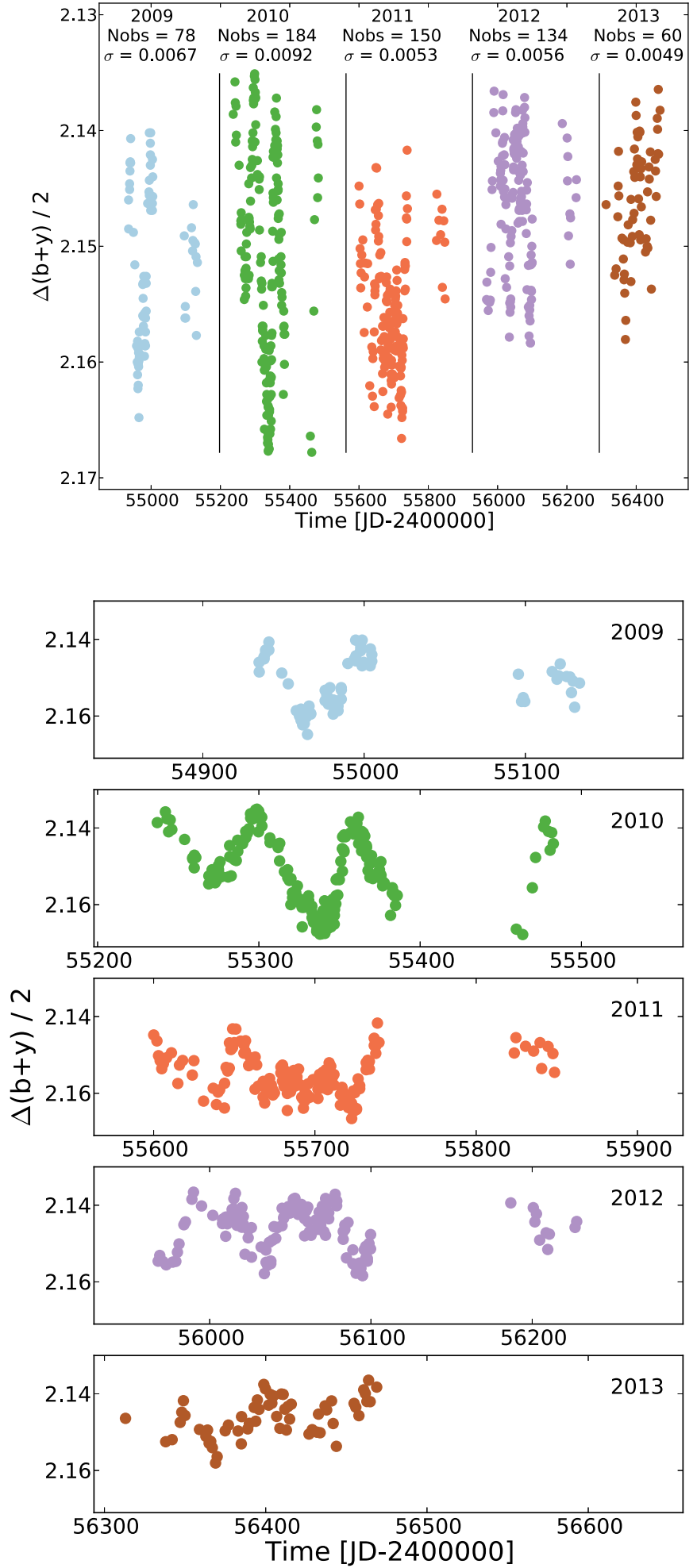

Figure 11. Photometric data taken of GJ 687 over 5 yr. The top panel shows the total data set with information regarding observations and standard deviation for each year. The bottom panel gives a closer look at the data separated by year.

(A color version of this figure is available in the online journal.)

that generates the periodogram peak. Departures from perfect periodicity are presumably caused by the evolution of the spot activity on the surface of the star.

Finally, we search for transits of GJ 687 b by first removing the spot variability from each of the yearly light curves. We do this by successively subtracting multiple frequencies from
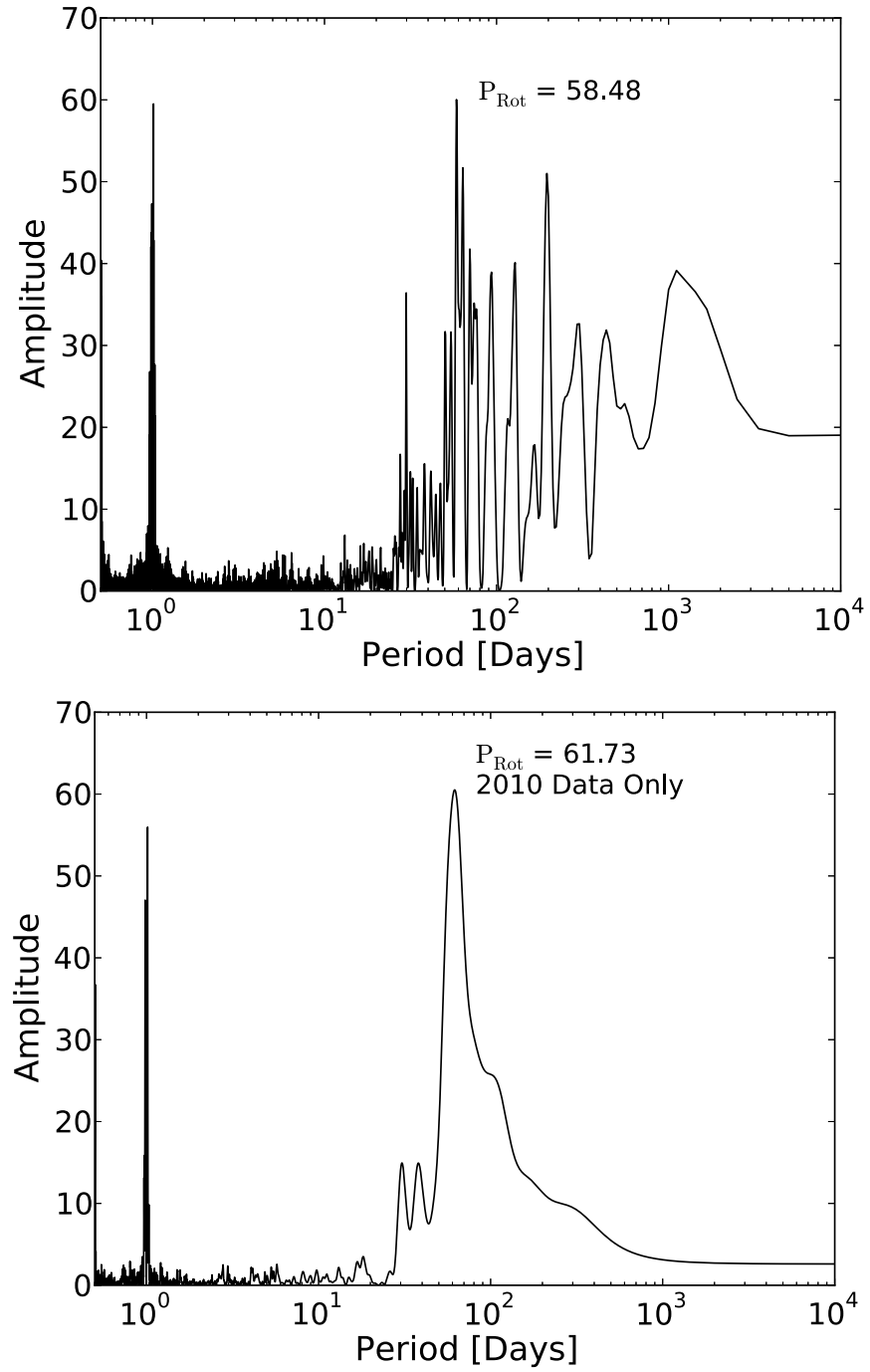

Figure 12. Lomb-Scargle periodogram of the photometric observations of GJ 687. In the combined data set, the maximum observed power occurs at 58.48 days (top panel). However when we consider only data obtained in 2010, where the rotational modulation is most clearly exhibited, we find that maximum power occurs at $P=61.73$ days (bottom panel). We identify this periodicity with the rotational period of the star.

each yearly light curve using the method described in Henry et al. (2001). We removed three to six frequencies from each light curve until each set of residuals approached the precision of a single observation. The residuals from all five observing seasons are plotted in the top panel of Figure 13, phased with the 38.14 day best-fit planetary orbital period and a time of mid transit computed from the orbital parameters. The vertical bar represents the $0.0022 \mathrm{mag}$ standard deviation of the residuals from their mean, very close to the measurement precision given above. A sine fit to the phased data gives a formal semiamplitude of just $0.00011 \pm 0.00012 \mathrm{mag}$. Since none of the frequencies removed from the yearly light curves were similar to the orbital frequency or its harmonics, this result limits any periodic brightness variability of the star on the observed $\mathrm{RV}$ period to a very small fraction of one millimagnitude (mmag). This rules out the possibility that the 38.14 day RV variations in GJ 687 are induced by stellar activity, as has been documented in somewhat more active stars, for instance, by Queloz et al. (2001), Paulson et al. (2004), and Boisse et al. (2012). Instead, this lack of photometric variability confirms 


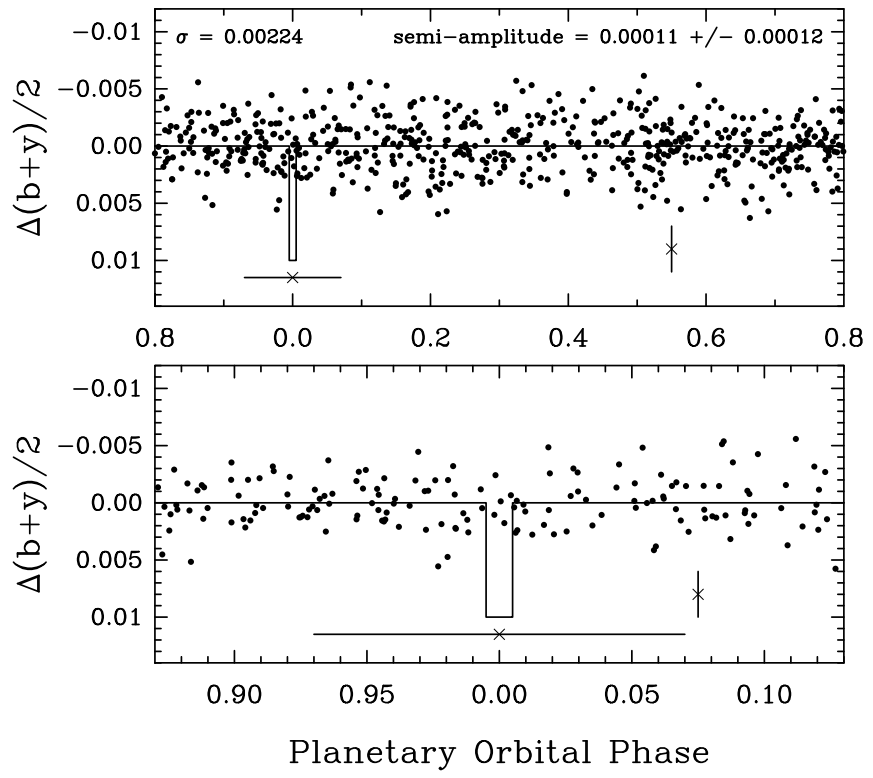

Figure 13. Top panel: filtered differential photometric measurements for Gliese 687 folded at the best-fit planetary period, $P=38.14$ days. A light curve model for a centrally transiting Neptune-sized planet is shown. The vertical error bar indicates the $0.002 \mathrm{mag}$ photometric precision. The horizontal error bar shows the $1 \sigma$ uncertainty on the time of a central transit. Bottom panel shows a magnified view of the folded photometric data in the vicinity of the predicted time of central transit.

Table 1

Stellar Parameters for Gliese 687

\begin{tabular}{lll}
\hline \hline Parameter & \multicolumn{1}{c}{ Value } & \multicolumn{1}{c}{ Reference } \\
\hline Spectral type & M3 V & Rojas-Ayala et al. (2012) \\
Mass $\left(M_{\odot}\right)$ & $0.413 \pm 0.041$ & Boyajian et al. (2012) \\
Radius $\left(R_{\odot}\right)$ & $0.4183 \pm 0.0070$ & Boyajian et al. (2012) \\
Luminosity $\left(L_{\odot}\right)$ & $0.0213 \pm 0.00023$ & Boyajian et al. (2012) \\
Distance $(\mathrm{pc})$ & $4.5 \pm 0.115$ & Rojas-Ayala et al. (2012) \\
$B-V$ & 1.5 & Simbad \\
$V$ mag. & 9.15 & Rojas-Ayala et al. (2012) \\
$J$ mag. & 5.335 & Cutri et al. (2003) \\
$H$ mag. & 4.77 & Cutri et al. (2003) \\
$K$ mag. & 4.548 & Cutri et al. (2003) \\
Avg. S-index & 0.811 & This work \\
$\sigma_{\text {S-index }}$ & 0.096 & This work \\
$P_{\text {rot }}($ days $)$ & $61.8 \pm 1.0$ & This work \\
$T_{\text {eff }}(\mathrm{K})$ & $3413 \pm 28$ & Boyajian et al. (2012) \\
\hline
\end{tabular}

that the RV variations in GJ 687 result from true planetary reflex motion.

The photometric observations within $\pm 0.13 P$ of mid-transit are replotted with an expanded scale in the bottom panel of Figure 13. The solid curve shows the predicted phase, depth (assuming Neptune-like density), and duration of a central transit, computed from the stellar radius in Table 1 and the orbital elements in Table 2. The horizontal error bar under the predicted transit time gives the $\pm 1 \sigma$ uncertainty in the timing of the transit. The photometric observations when filtered using the Henry et al. (2001) procedure described above, and when folded at the $P=38.14$ day best-fit period for the planet, give no indication that transits occur. We note, however, that the Markov Chain models generate a five-day window for possible transits, and so a more conservative approach is also warranted. In Figure 14, we plot the unfiltered photometric data, indicating the range of photometric points that potentially could have been affected by transits were they to occur. Because of uncertainties

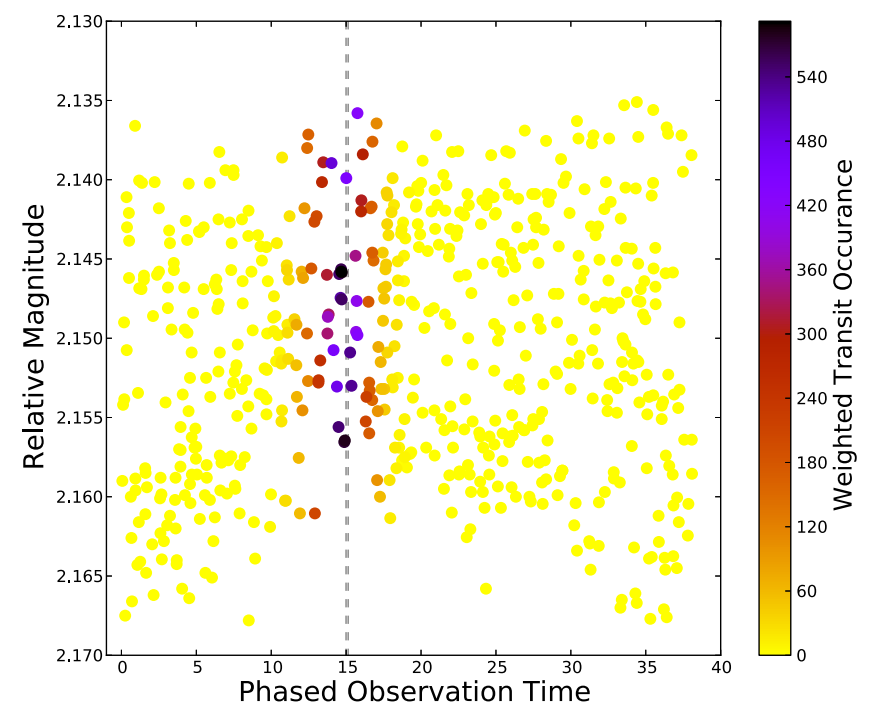

Figure 14. For each of the 10,402 potential systems in our Markov chain, we check the predicted transit times against our photometric observations. If a photometric data point lies within the transit window of a particular member of the Markov chain, we assign a value to that point which is cosine-weighted by its distance from the predicted time of central transit. The sum of these values is mapped onto the color of the points in the diagram. The phase of the points, as well as the vertical gray bar spanning the predicted $3 \mathrm{hr}$ central transit duration are for our best fit model given in Table 2 .

(A color version of this figure is available in the online journal.)

Table 2

One-planet Model for the GJ 687 System

\begin{tabular}{lll}
\hline \hline Parameter & \multicolumn{1}{c}{ Best fit } & \multicolumn{1}{c}{ Errors } \\
\hline Period (days) & 38.14 & $(0.015)$ \\
Mass $\left(M_{J}\right)$ & 0.058 & $(0.007)$ \\
Mass $\left(M_{\oplus}\right)$ & 18.394 & $(2.167)$ \\
Mean anomaly (deg) & 234.62 & $(87.962)$ \\
Eccentricity & 0.04 & $(0.076)$ \\
Longitude of periastron $(\mathrm{deg})$ & 359.43 & $(120.543)$ \\
Semi-major axis (AU) & 0.16353 & $(0.000043)$ \\
Time of periastron (JD) & 2450579.11 & $(9.32)$ \\
RV half amplitude (m s $\left.{ }^{-1}\right)$ & 6.43 & $(0.769)$ \\
First observation epoch (JD) & 2450603.97 & \\
Velocity offsets & & $(1.68)$ \\
Keck/HIRES & $0.64 \mathrm{~m} \mathrm{~s}^{-1}$ & $(0.98)$ \\
APF/Levy & $-1.71 \mathrm{~m} \mathrm{~s}^{-1}$ & \\
HET & $1.27 \mathrm{~m} \mathrm{~s}^{-1}$ & \\
$\chi^{2}$ & 18.55 & \\
rms & & \\
Keck/HIRES & $6.62 \mathrm{~m} \mathrm{~s}^{-1}$ & \\
APF/Levy & $3.95 \mathrm{~m} \mathrm{~s}^{-1}$ & \\
HET & $2.44 \mathrm{~m} \mathrm{~s}^{-1}$ & \\
Jitter & $5.93 \mathrm{~m} \mathrm{~s}^{-1}$ & \\
\hline
\end{tabular}

Notes. All elements are defined at epoch JD $=2450603.97$. Uncertainties are reported in parentheses.

in the orbit, the potential transit duration, the potential size of the planet, and the error in the photometric filtering, we recommend that continued photometric monitoring be carried out to confirm that transits do not occur.

\section{METALLICITY}

Gliese 687 appears to have a slightly sub-solar metallicity. Rojas-Ayala et al. (2012) use Na I, Ca I, and $\mathrm{H}_{2} \mathrm{O}-\mathrm{K} 2$ calibrations to estimate $[\mathrm{Fe} / \mathrm{H}]=-0.09$ for Gliese 687 , whereas the 
M dwarf metallicity calibration of Schlaufman \& Laughlin 2010 yields a value $[\mathrm{Fe} / \mathrm{H}]=-0.02$.

The connection between the detectable presence of a giant extrasolar planet and the metallicity of the host star was noticed soon after the first extrasolar planets were detected (Gonzalez 1997), and has been studied in many previous works, see, e.g., Fischer \& Valenti (2005); Sousa et al. (2011). For M dwarfs, recent work, such as that by Neves et al. (2013), suggests that the giant planet stellar metallicity correlation holds robustly for M dwarf primaries, but that for planets with mass, $M_{\mathrm{p}} \lesssim 20 M_{\oplus}$, no correlation is found with host star metallicity, and indeed, Neves et al. (2013) and Jenkins et al. (2013) report a hint of anti-correlation between the presence of a low-mass planet and host star $[\mathrm{Fe} / \mathrm{H}]$. Our detection of a Neptune-mass companion to Gliese 687, and our Lick-Carnegie database of Doppler measurements of $\mathrm{M}$ dwarf stars provides an opportunity to revisit this topic.

Our database of RV observations taken at the Keck Telescope contains 142 M-type stars with the necessary spectral information to assess metallicity, 17 of which are known to host planets published in the peer-reviewed literature. We break the planethosting stars into two subgroups based on their masses-stars with $M \sin (i)$ planets less than $30 M_{\text {Earth }}$ are described as Neptune hosting while stars with $M \sin (i)$ planets greater than $30 M_{\text {Earth }}$ are listed as Jupiter hosting. We replicate the procedure of Schlaufman \& Laughlin (2010) and examine how horizontal distance from a field $\mathrm{M}$ dwarf main sequence in a $M_{K_{s}}$ versus $\left(V-K_{s}\right)$ CMD correlates with metallicity, as noted, e.g., by Baraffe et al. (1998). The top panel of Figure 15 displays all of the Lick-Carnegie survey M dwarf stars plotted in $M_{K_{s}}$ versus $\left(V-K_{S}\right)$, with gray dots denoting survey stars without known planets, red dots denoting survey stars that host "Neptune-mass" planets and blue dots representing the survey stars that host "Jupiter-mass" planets. It can be seen that most planet hosting stars fall to the right of the field $\mathrm{M}$ dwarf main sequence presented in Johnson \& Apps (2009) (black line), which is taken to be a $[\mathrm{Fe} / \mathrm{H}]=-0.17$ isometallicity contour in this CMD. In order to quantify the likelihood that a star's horizontal distance from the isometallicity contour is related to its propensity to host planets, we compare the distances for our actual planet-hosting stars with randomly drawn samples from the collection of $\mathrm{M}$ dwarfs in the survey.

We characterize the position of each $\mathrm{M}$ dwarf by obtaining $V$-band and $K_{s}$ photometry and then using them to calculate the distance statistic $\Sigma$ :

$$
\Sigma=\sum_{i=1}^{n}\left(V-K_{s}\right)_{i}-\left(V-K_{s}\right)_{\text {iso }} .
$$

To determine if the $\Sigma$ of our known planet hosting subgroups is significant or, alternatively, if it could be produced by chance, we make use of a Monte Carlo simulation that calculates the cumulative sample distance of survey $M$ dwarfs from the field $\mathrm{M}$ dwarf main sequence presented by Johnson \& Apps (2009). For the simulation, we randomly select a subset of M dwarfs from the Lick-Carnegie field star list, setting the sample size equal to the number of $\mathrm{M}$ dwarfs known to host either Jovian or Neptune mass planets. Then we compute the cumulative horizontal distance of those stars from the field M dwarf MS, where stars to the right of the MS add their distance to the sum and stars to the left of the MS subtract their distance. We repeat this process 10,000 times to determine the distribution of cumulative horizontal distances from the MS
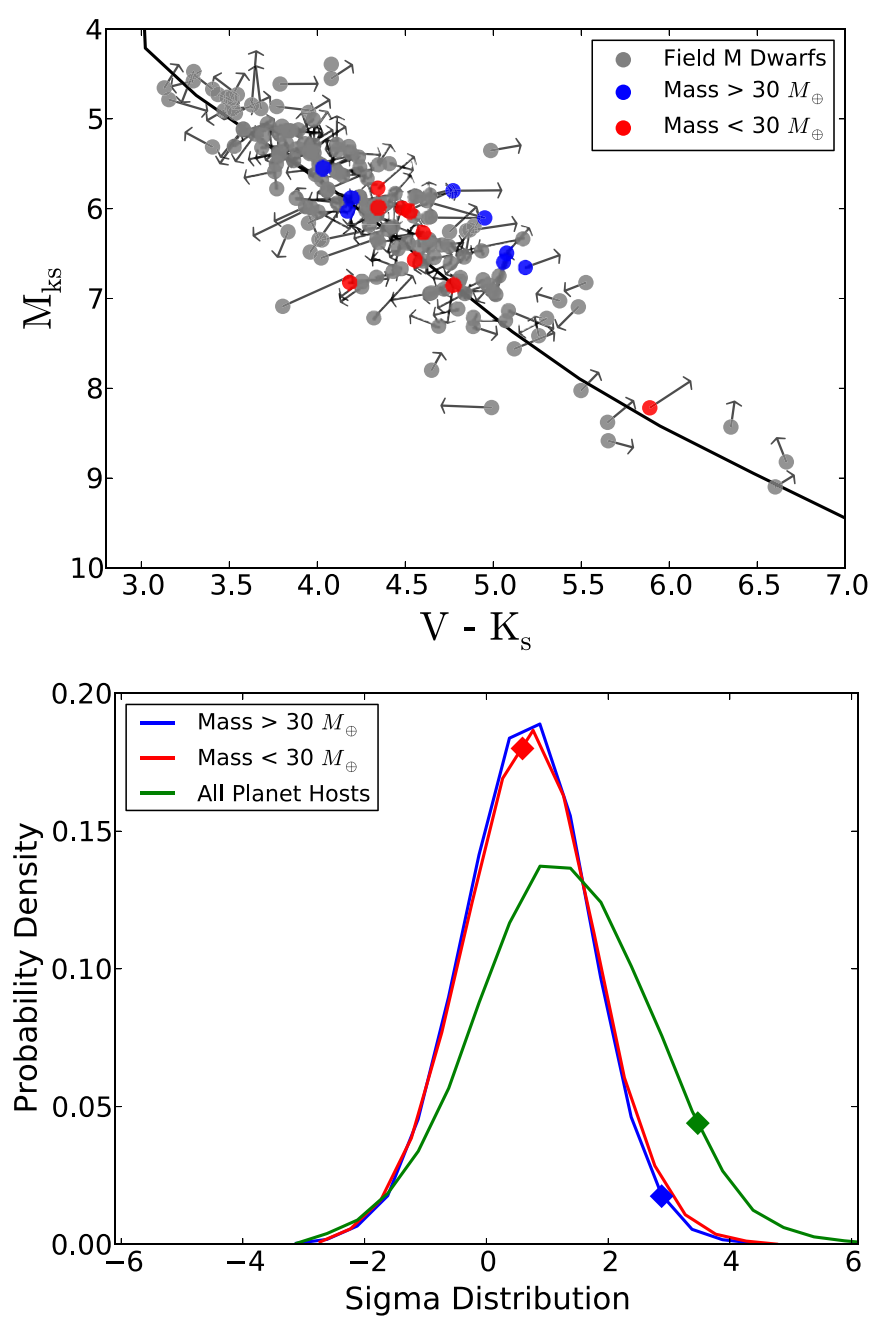

Figure 15. Top panel: location of the $142 \mathrm{M}$ dwarfs from the Lick-Carnegie radial velocity survey. Stars known to host Jupiter-mass planets are plotted in blue, those known to host twice-Neptune $M \sin (i)$ (or smaller) planets are plotted in red, and non-planet hosting survey $M$ dwarfs are plotted in gray. The field M dwarf main sequence from JA09 is shown as a black line and the arrows affixed to each point represent that survey star's proper motion. Bottom panel: distributions generated via Monte Carlo simulations of the cumulative sample distance of field M dwarfs ( $\Sigma$ as defined in Equation (2)) from the M dwarf main sequence used by Johnson \& Apps (2009). The points plotted on top of each curve in the bottom panel represent the actual cumulative distance from the MS for our planet hosting and field star samples.

(A color version of this figure is available in the online journal.)

given no correlation between whether the star hosts an exoplanet and its location in the $\left(V-K_{s}\right)-\left(M_{K_{s}}\right)$ CMD.

Our results show that for hosts of Jupiter mass planets, $\Sigma=$ 2.359 , which corresponds to a probability of $p=0.053_{-0.04}^{+0.13}$ that the stars' cumulative distance from the isometallicity contour occurred by chance. For the Neptune hosts, we find that $\Sigma=1.113$ leading to $p=0.452_{-0.23}^{+0.24}$, and for the combination of all planet hosts, we obtain $\Sigma=3.473$ or $p=0.0775_{-0.05}^{+0.09}$. The distributions resulting from the Monte Carlo simulation and the locations of the actual planet hosting stars $\Sigma$ values are displayed in the bottom panel of Figure 15. The points plotted on top of each curve in Figure 15 represent the actual cumulative distance of our planet hosting star samples from the field M dwarf MS. Our results thus indicate that the planet-metallicity correlation is robust for $\mathrm{M}$ dwarf hosts of planets with $M>30 M_{\oplus}$, but that at smaller masses there is, at present, no evidence a correlation exists. 


\section{PLANET RECOVERY}

The Lick-Carnegie exoplanet survey and its predecessors have carried out a long-term monitoring program of the brightest M dwarf stars in the sky. Our database of observations contains 159 stars that have more than 10 observations apiece, and which, additionally, have median internal uncertainty $\sigma<10 \mathrm{~m} \mathrm{~s}^{-1}$. Within this group, there is a subset with extensive data sets. For example, 11 stars have $N>100$ observations and median internal uncertainties $\sigma<13 \mathrm{~m} \mathrm{~s}^{-1}$. A question of substantial interest, therefore, is the degree to which the observations taken to date have probed the true aggregate of planetary companions to the M dwarf stars in our survey.

The effort required to obtain the existing data has been substantial. Among the M-type stars alone, our database contains a total of 5,468 velocity measurements from Keck I, totaling $2,579,862 \mathrm{~s}$ (29.86 days) of on-sky integration. Overheads, including the acquisition of high $\mathrm{S} / \mathrm{N}$ spectra, $\mathrm{CCD}$ readout time, and weather losses, add materially to this time investment. Furthermore, the distribution of total observing time allotted to the stars on the list has been highly uneven. Targets such as Gliese 436 and Gliese 876, which harbor planetary systems of particular interest, have received much more attention than the typical red dwarf in the survey. For example, Gliese 436 has 148 observations and Gliese 876 has 204 observations obtained with the Keck Telescope. The stars themselves also exhibit a range of chromospheric activity levels. The resulting star-to-star dispersion in "stellar jitter" (tantamount to a measurement uncertainty, $\sigma_{\mathrm{jit}}$ ) complicates the evaluation of threshold levels for $M \sin (i)$ as a function of orbital period to which planetary companions can be excluded.

There are a variety of approaches to the measurement of FAPs in the context of spectral analysis of unevenly sampled data; see, e.g., Baluev (2012) for a recent discussion. A very simple approach is described by Press et al. (1992). For a Gaussian random variable,$^{5}$ the probability distribution for obtaining a peak at frequency $\omega$ of Lomb-normalized power (Scargle 1982), $P_{\mathrm{N}}(\omega)$, is exponential with unit mean. If a data set drawn from measurements of a white noise (Gaussian) distribution supports measurement of $M$ independent frequencies, the probability that no peak exceeds power $z$ (the FAP) is $\mathcal{P}\left(P_{\mathrm{N}}>z\right)=$ $1-\left(1-\exp ^{-z}\right)^{M}$.

We adopt a FAP of $10^{-4}$, calculated with the above method (and using Monte-Carlo simulations to determine $M$ ) as the generic threshold for attributing a given planetary signal to a given data set. With this detectability threshold, we use the Systemic Console 2.0 software package (Meschiari et al. 2012) to determine the number of readily detectable planets in our M dwarf data set. A "readily detectable" planet generates a signal that can be isolated algorithmically (and automatically) by straightforward periodogram analysis and Levenberg-Marquardt minimization. The results of this exercise are shown in Table 3, which locates signals corresponding to 19 previously published planets orbiting 14 separate M dwarf primaries. The table lists both the published planetary parameters from exoplanets.org (Wright et al. 2011) and the parameters calculated by our search. Other than Gliese $667 \mathrm{C}$, there are no stars on our 159 star list for which a planet has been published by another group, and for which the automated algorithm

\footnotetext{
5 Clearly, the generating function for typical radial velocity data sets has non-Gaussian (and unknown) error. False alarm probabilities must therefore be treated with great caution when evaluating the existence of a planet with $K \gtrsim \sigma_{\text {unc. }}$.
}

Table 3

Planets Found from 179 Local M Dwarf Stars

\begin{tabular}{|c|c|c|c|c|c|}
\hline Star & Planet & Source & $\begin{array}{c}\text { Per } \\
\text { (days) }\end{array}$ & $M_{\text {Jup }}$ & Ecc \\
\hline \multirow[t]{2}{*}{ HD 285968} & GJ 176 b & Calc & 8.775 & 0.031 & 0.0 \\
\hline & GJ 176 b & Pub & 8.7832 & 0.026 & 0.0 \\
\hline \multirow[t]{2}{*}{ HIP 22627} & GJ 179 b & Calc & 2249.994 & 0.829 & 0.197 \\
\hline & GJ 179 b & Pub & 2288 & 0.824 & 0.210 \\
\hline \multirow[t]{2}{*}{ GJ 1214} & GJ 1214 b & Calc & 2.732 & 0.024 & 0.11 \\
\hline & GJ 1214 b & Pub & 1.580 & 0.0204 & 0.0 \\
\hline \multirow[t]{2}{*}{ GL 317} & GL 317 b & Calc & 692.965 & 1.164 & 0.0 \\
\hline & GL $317 \mathrm{~b}$ & Pub & 692.9 & 1.18 & 0.193 \\
\hline GL 382 & GL 382 & Calc & 36.380 & 0.071 & 0.0 \\
\hline GL 388 & GL 388 & Calc & 2.226 & 0.143 & 0.06 \\
\hline \multirow[t]{2}{*}{ GL 433} & GL 433 b & Calc & 7.3699 & 0.016 & 0.2 \\
\hline & GL 433 b & Pub & 7.371 & 0.0182 & 0.080 \\
\hline GL 625 & GL 625 & Calc & 21.316 & 0.032 & 0.33 \\
\hline \multirow[t]{3}{*}{ GL 876} & GJ 876 b & Calc & 61.033 & 1.924 & 0.0 \\
\hline & GJ 876 c & Calc & 30.228 & 0.636 & 0.01 \\
\hline & GJ 876 d & Calc & 15.042 & 0.117 & 0.05 \\
\hline \multirow[t]{4}{*}{ GL 876} & GJ 876 b & Pub & 61.117 & 1.95 & 0.032 \\
\hline & GJ 876 c & Pub & 30.088 & 0.612 & 0.26 \\
\hline & GJ $876 \mathrm{~d}$ & Pub & 1.938 & 0.018 & 0.207 \\
\hline & GJ 876 e & Pub & 124.26 & 0.0392 & 0.055 \\
\hline \multirow[t]{2}{*}{ HIP 109388} & GJ 849 b & Calc & 2001.096 & 1.049 & 0.0 \\
\hline & GJ 849 b & Pub & 1880 & 0.83 & 0.040 \\
\hline \multirow[t]{2}{*}{ HIP 57050} & HIP 57050 b & Calc & 41.373 & 0.267 & 0.291 \\
\hline & HIP $57050 \mathrm{~b}$ & Pub & 41.397 & 0.298 & 0.314 \\
\hline \multirow[t]{2}{*}{ HIP 57087} & GJ 436 b & Calc & 2.644 & 0.067 & 0.149 \\
\hline & GJ 436 b & Pub & 2.644 & 0.0737 & 0.15 \\
\hline HIP 70890 & HIP 70890 & Calc & 364.742 & 0.021 & 0.1 \\
\hline \multirow[t]{3}{*}{ HIP 74995} & GJ 581 b & Calc & 5.368 & 0.048 & 0.0 \\
\hline & GJ 581 c & Calc & 12.918 & 0.017 & 0.156 \\
\hline & GJ $581 \mathrm{~d}$ & Calc & 68.689 & 0.017 & 0.4 \\
\hline \multirow[t]{3}{*}{ HIP 74995} & GJ 581 b & Pub & 5.369 & 0.0499 & 0.031 \\
\hline & GJ 581 c & Pub & 12.918 & 0.0168 & 0.070 \\
\hline & GJ $581 \mathrm{~d}$ & Pub & 66.640 & 0.0191 & 0.250 \\
\hline \multirow[t]{2}{*}{ HIP 83043} & GJ 649 b & Calc & 592.764 & 0.267 & 0.33 \\
\hline & GJ 649 b & Pub & 598.3 & 0.325 & 0.3 \\
\hline \multirow[t]{2}{*}{ HIP 85523} & GJ 674 b & Calc & 4.691 & 0.034 & 0.19 \\
\hline & GJ 674 b & Pub & 4.694 & 0.035 & 0.2 \\
\hline \multirow[t]{2}{*}{ HIP 109388} & GJ 849 b & Calc & 2001.099 & 1.049 & 0.14 \\
\hline & GJ 849 b & Pub & 1880 & 0.83 & 0.040 \\
\hline \multirow[t]{2}{*}{ HD 204961} & GJ 832 b & Calc & 3440.923 & 0.639 & 0.12 \\
\hline & GJ 832 b & Pub & 3420 & 0.644 & 0.12 \\
\hline
\end{tabular}

finds no planets. Regarding GJ 667C, 40 observations have been made at Keck, and these were used in a characterization of the GJ 667C system (Anglada-Escudé et al. 2012), however the peak planetary signal for this set fell below our FAP threshold when utilizing only the Keck data. The bright planet-hosting red dwarfs Gliese 832, 3634, and 3470 all have declinations that are too far south to be observed from Mauna Kea, and HIP 79431 (R.A. $16^{\mathrm{h}} 12^{\mathrm{m}} 41^{\mathrm{s}} .77$, decl. $-18^{\circ} 52^{\prime} 31^{\prime \prime} .8$ ) is not on the list of $\mathrm{M}$ dwarfs being monitored at Keck.

The Kepler mission's photometric data have been used to infer that small planets orbiting $\mathrm{M}$ dwarfs are very common. For example, Dressing \& Charbonneau (2013) find an occurrence rate of 0.9 planets per star in the range $0.5 R_{\oplus}<R_{\mathrm{p}}<4 R_{\oplus}$ 

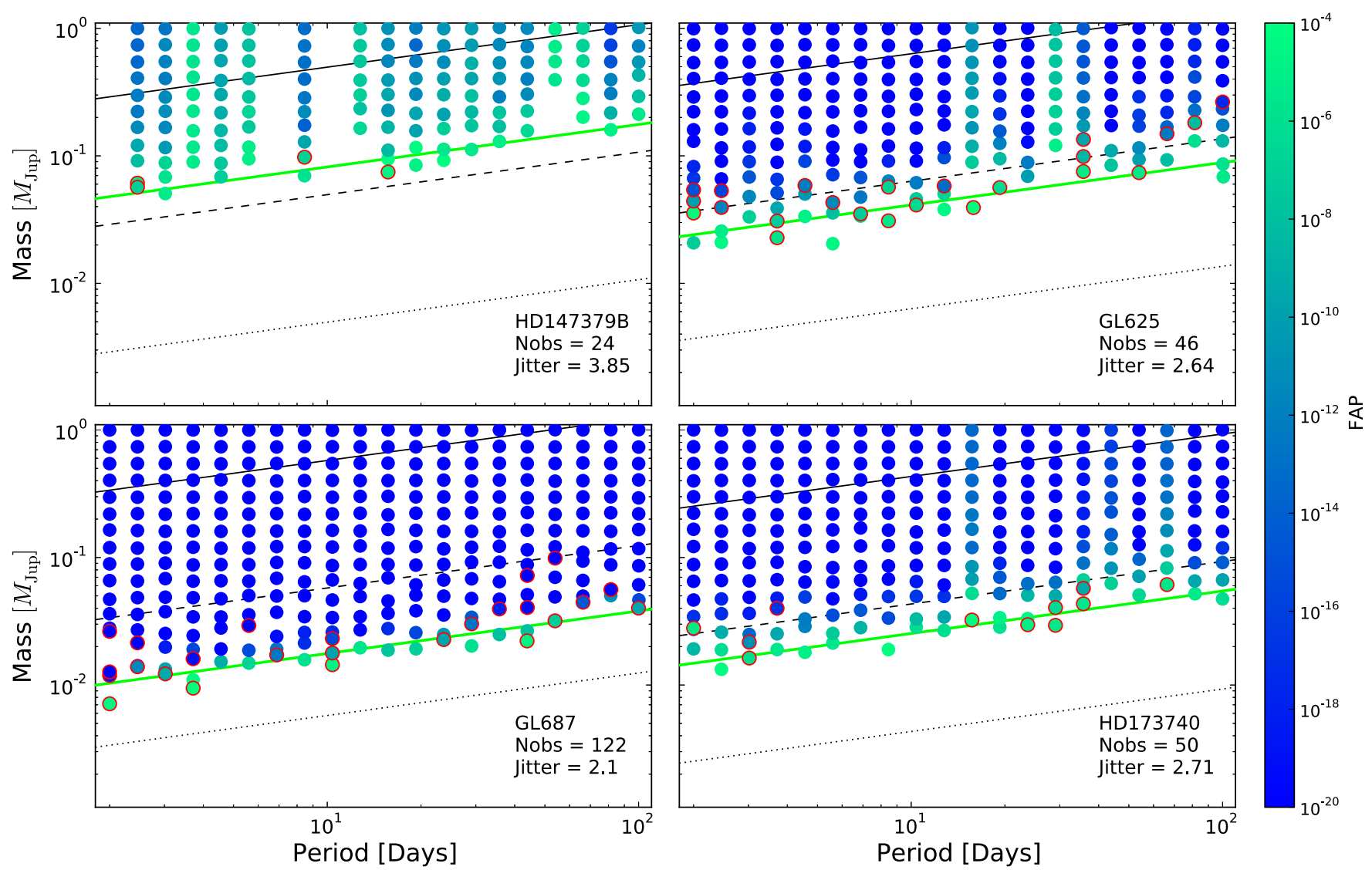

Figure 16. Example plots of our synthetic planet recovery around four $M$ dwarf stars. The points represent planets our algorithm found, colored by the false alarm probability for the initial detection. The black lines from bottom to top show radial velocity half amplitudes $(K)$ of 1,10 , and $100 \mathrm{~m} \mathrm{~s}^{-1}$. The green line is our minimum detectable $K$ value.

(A color version of this figure is available in the online journal.)

with $P<50$ days. Given the existence of this large number of small-radius planets, it is of interest to make a quantitative analysis of how deep into the expected population of superEarth-type planets suggested by the Kepler mission the Keck Radial Velocity Survey has probed. To answer this question, we have created synthetic RV data sets that contain test planets, and which conform with the timestamps, the internal measurement uncertainties, and the stellar properties (namely mass) for all $104 \mathrm{M}$ dwarfs under surveillance at Keck with at least $20 \mathrm{RV}$ observations. To address the error source arising from stellar jitter, $\sigma_{\mathrm{jit}}$, we use the median value provided in Wright (2005) of $3.9 \mathrm{~m} \mathrm{~s}^{-1}$ as the expected level of $\sigma_{\text {jit }}$ for our M dwarf stars. This value is then added in quadrature with the internal uncertainty and applied to the synthetic data set to create a more accurate representation of the system.

For each of the 104 stars in the Keck survey, we have created 400 synthetic data sets. Each set contains a single planet. The planets are evenly spaced in log period from 2 to 100 days, and evenly spaced in $\log$ mass from $1 M_{\oplus}$ to $1 M_{\text {Jup }}$. We assign a circular orbit to these test planets and assume $i=90^{\circ}$ and $\Omega=$ $0^{\circ}$ in each case. We then calculate the RV each of these planets would induce on a parent star. A Gaussian distribution with $\sigma^{2}=\sigma_{\text {internal }}{ }^{2}+\sigma_{\text {jitter }}{ }^{2}$ is used to perturb the predicted $\mathrm{RV}$ value.

Each of the $104 \times 400$ synthetic systems is passed to the planet search algorithm. Figure 16 shows examples of the returned planets for 4 of the 104 stars in this experiment including the star of main interest here, GJ 687. In Figure 16 the black lines from bottom to top represent constant $K=(2 \pi G / P)^{1 / 3} M_{\mathrm{P}} M_{\star}{ }^{-2 / 3}$ values of 1,10 , and $100 \mathrm{~m} \mathrm{~s}^{-1}$ respectively. As expected, the detectablity thresholds lie roughly along lines of constant $K$. To determine the smallest $K$ value we could reliably detect for each star, we find the smallest value of $K$ for which a planet was found for at least $50 \%$ of the chosen periods. This median value generates the green lines seen in the figure. The top panel of Figure 17 shows these minimum $K$ values for each of the 104 stars that we tested. For clarity, the stars in this figure have been ordered by increasing minimum $K$, and are colored by the number of observations we have for each.

If a test planet lies in its star's habitable zone, (defined as the semi-major axis at which the flux received by the planet is the solar constant received at Earth) we can ask how large the planet needs to be to be detectable by our RV survey. Figure 17 shows these threshold masses for each of the $104 \mathrm{M}$ dwarf stars which we analyzed. These stars maintain the ordering from the top panel, but now have been colored by the mass of the parent star. We see that while the Keck survey has probed substantially into the regime occupied by Neptune-mass planets, it has not made significant inroads into the super-Earth regime for periods that are of astrobiological interest.

\section{DISCUSSION}

GJ 687 is the second planetary system to be detected using data from the APF telescope, with the first being HD 141399 b,c,d, and e (Vogt et al. 2014a). APF has successfully navigated its commissioning stage, and, since Q2 2013, it has routinely acquired science-quality data that presents sub-m/s precision on known RV standard stars (Vogt et al. 2014b). In recent months, 

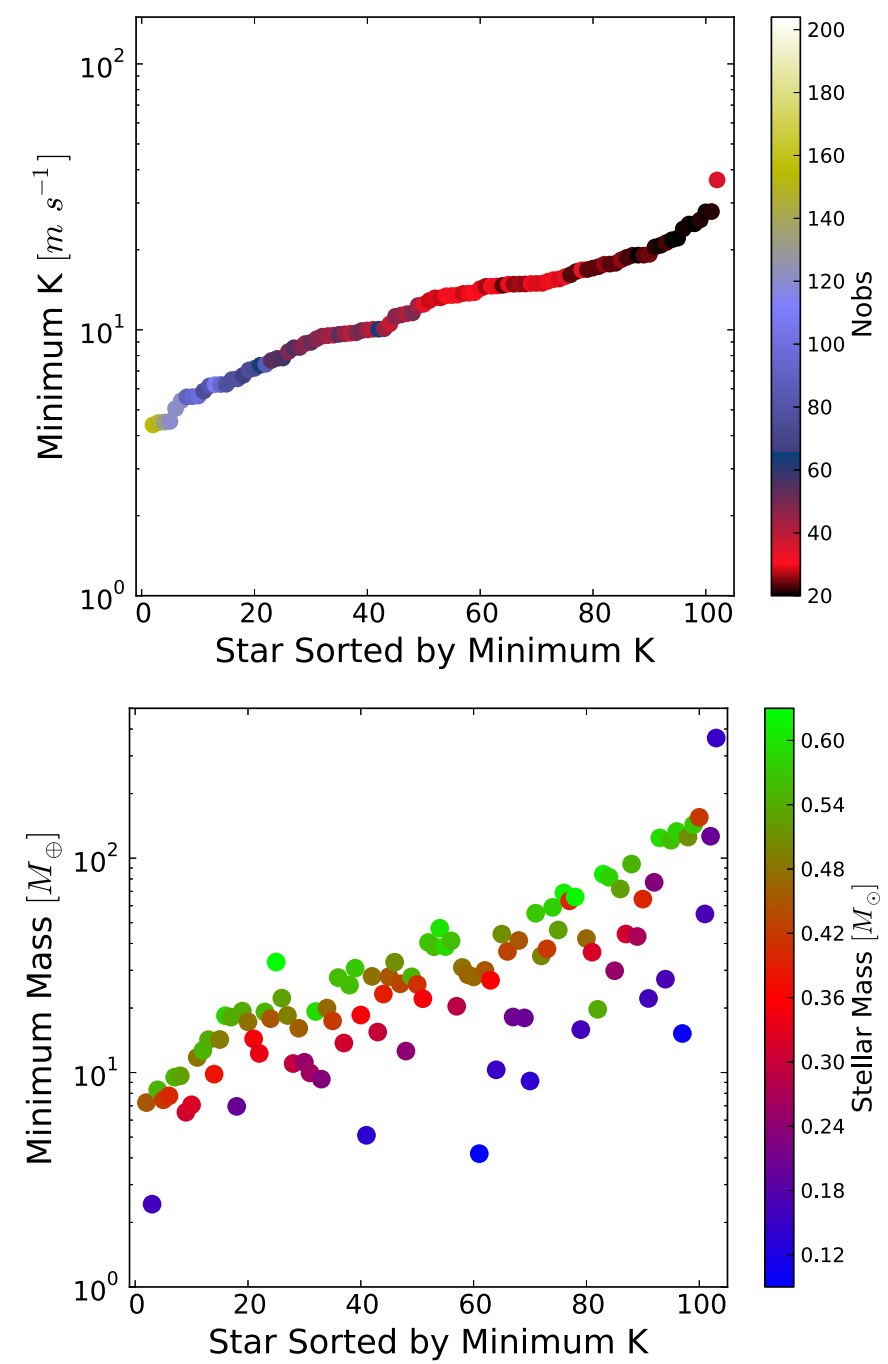

Figure 17. Top panel: the minimum detectable $K$ value for each star in our M dwarf collection. Bottom panel: assuming a minimum detectable $K$ for each $M$ dwarf star, if a planet was orbiting in that star's habitable zone, this is the minimum mass that planet could have and still be recovered by our method.

(A color version of this figure is available in the online journal.)

the degree of automation for APF has increased substantially. The facility currently works autonomously through an entire night's operations, calibration, and observing program. The APF and its accompanying high-resolution Levy spectrograph together form a dedicated, cost-effective, ground-based precision $\mathrm{RV}$ facility that is capable of detecting terrestrial-mass planets at distances from their parent stars at which surface liquid water could potentially be present.

Unlike other highly successful RV facilities, the APF uses neither image scrambling nor image slicing. With a peak efficiency of $15 \%$ and typical spectral resolutions of $R \sim 110,000$, the APF represents a critical new resource in the global quest to detect extrasolar planets. Initial speed comparisons indicate that in order to match the signal-to-noise acquired using the Keck telescope/HIRES Spectrograph combination, the APF needs only a factor of 6 increase in observing time. Since the amortized cost of a night on Keck is $\sim 77$ times more expensive than a night on the APF, and because $80 \%$ of the APF's nights are reserved for exoplanetary work, the APF (with its sub-m/s precision and dedicated nightly cadence abilities) will likely provide key contributions to exoplanet detection and characterization in the coming years.

Gliese 687 b's RV half-amplitude, $K=6.4 \pm 0.5 \mathrm{~m} \mathrm{~s}^{-1}$, is substantially greater than the current state-of-the-art detection threshold for low-mass planets. The lowest measured value for $K$ in the catalog of Doppler-detected extrasolar planets 6 stands at $K=0.51 \mathrm{~m} \mathrm{~s}^{-1}$ (Dumusque et al. 2012). On the other hand, Gliese 687's status as one of the nearest stars to the Sun imbues it with a great deal of intrinsic interest. In our view, the relatively recent date for Gliese 687 b's detection can be attributed both to the substantial amount of stellar-generated RV noise (as evidenced by Figures 3 and 5), but also to its location in Draco, high in the Northern Sky, where APF, along with HARPS North, are the only facilities that can routinely observe at sub-1 $\mathrm{m} \mathrm{s}^{-1}$ precision. (As evidenced by the data in this paper, Keck can observe at these high declinations, but at significantly higher expense in comparison to stars lying closer to the celestial equator.)

Indeed, Gliese 687's stellar coordinates (R.A. $17^{\mathrm{h}} 36^{\mathrm{m}}$, decl. $+68^{\circ}$ ) place it very close to the north ecliptic pole, located at R.A. $=18^{\mathrm{h}}$, decl. $=+66^{\circ}$. This location flags it as a star of potentially great importance for the forthcoming NASA TESS Mission. As currently envisioned (and as currently funded), TESS is a two-year, all-sky photometric survey to be carried out by a spacecraft in a 27 day $\mathrm{P} / 2$ lunary resonant orbit. TESS will photometrically monitor $\sim 500,000$ bright stars with a $<60$ ppm $1 \mathrm{hr}$ systematic error floor. (For reference, a central transit of the Sun by the Earth produces an $86 \mathrm{ppm}$ transit depth.) The northern ecliptic hemisphere will be mapped during the first year of the mission via a sequence of 13 sectors with 27 days of continuous observation per sector. These sectors overlap at the north ecliptic pole, and create an area of $\sim 1000 \mathrm{deg}^{2}$ (1/50th of the sky) for which photometric baselines will approach 365 days. Gliese 687 lies at the center of this TESS "overlap zone" (which also coincides with JWST's continuous viewing zone). Because much longer time series are produced in the overlap zone, the highest-value transiting planets found by the mission will emerge from this part of the sky (along with the sister segment covering the south ecliptic pole).

As mentioned above, however, the TESS overlap zone has received relatively little attention from the highest-precision Doppler surveys. About 10,000 target stars from the TESS Dwarf Star Catalog, all with $V<12$, are present in the overlap zone. This, of course, is far too many stars to survey with Doppler RV, but there appears to be substantial value inherent in monitoring the brightest, nearest, and quietest members of the cohort of TESS overlap stars. The latest estimates (Mayor et al. 2009, 2011; Batalha et al. 2013; Petigura et al. 2013) suggest that $\sim 50 \%$ of main sequence stars in the solar vicinity harbor $M>M_{\oplus}$ planets with $P<100$ days. Assuming a uniform distribution in period between 5 and 100 days, the average transit probability for these planets is $\mathcal{P} \sim 2.5 \%$, suggesting that of order $\mathcal{N} \sim 0.5 \times 0.025 \times 10,000 \sim 125$ low-mass transiting planets (and systems of transiting planets) will be detected by TESS within the overlap zone. Of these, a small handful, of order 5 systems total (and perhaps, with probability $\mathcal{P}_{\text {transit }}=1.2 \%$, including Gliese $687 \mathrm{~b}$ ) will garner by far the most attention from follow-up platforms such as JWST, due to their having optimally bright parent stars.

Our detection of Gliese $687 \mathrm{~b}$ suggests that by starting now, with a systematic program of Doppler observations of a target

\footnotetext{
6 www.exoplanets.org
} 
list of $\sim 200$ carefully vetted $\mathrm{G}, \mathrm{K}$ and $\mathrm{M}$ dwarf stars with $V \sim 7.5$ to $V \sim 10.5$ in the $1000 \mathrm{deg}^{2}$ TESS overlap zone, APF can ensure that a precise multi-year Doppler velocity time series will exist for the most important TESS planet host stars at the moment their transiting planets are discovered.

This research has made use of the Exoplanet Orbit Database and the Exoplanet Data Explorer at exoplanets.org. G.L. acknowledges support from the NASA Astrobiology Institute through a cooperative agreement between NASA Ames Research Center and the University of California at Santa Cruz. S.S.V. gratefully acknowledges support from NSF grants AST0307493 and AST-0908870. R.P.B. gratefully acknowledges support from NASA OSS grant NNX07AR40G, the NASA Keck PI program, and from the Carnegie Institution of Washington. S.M. acknowledges support from the W. J. McDonald Postdoctoral Fellowship. G.W.H. acknowledges support from NASA, NSF, Tennessee State University, and the State of Tennessee through its Centers of Excellence program. The work herein is based on observations obtained at the W. M. Keck Observatory, which is operated jointly by the University of California and the California Institute of Technology, and we thank the UC-Keck, UH, and NASA-Keck Time Assignment Committees for their support. This research has made use of the Keck Observatory Archive (KOA), which is operated by the W. M. Keck Observatory and the NASA Exoplanet Science Institute (NExScI), under contract with the National Aeronautics and Space Administration. We thank those who collected the data in the KOA including: D. Fischer, G. Marcy, J. Winn, W. Boruki, G. Bakos, A. Howard, and T. Bida. We also wish to extend our special thanks to those of Hawaiian ancestry on whose sacred mountain of Mauna Kea we are privileged to be guests. Without their generous hospitality, the Keck observations presented herein would not have been possible. This research has made use of the SIMBAD database, operated at CDS, Strasbourg, France. This paper was produced using ${ }^{B} A^{M}$.

Facilities: Keck:I (HIRES), APF (Levy Spectrometer), HET (High-Resolution-Spectrograph)

\section{APPENDIX \\ Doppler Radial Velocity Observations ${ }^{7}$}

Tables 4-6 present the collection of RV data used in the detection of GL687. All data presented here have been corrected to the solar system's barycentric reference frame.

\footnotetext{
7 Observations are corrected to the solar system's barycentric reference frame.
}

Table 4

(Continued)

\begin{tabular}{|c|c|c|}
\hline JD & $\begin{array}{c}\mathrm{RV} \\
\left(\mathrm{m} \mathrm{s}^{-1}\right)\end{array}$ & $\begin{array}{c}\text { Uncertainty } \\
\left(\mathrm{m} \mathrm{s}^{-1}\right)\end{array}$ \\
\hline 2452007.029 & -3.280 & 1.970 \\
\hline 2452009.077 & -7.440 & 2.020 \\
\hline 2452061.913 & 7.450 & 2.040 \\
\hline 2452062.935 & 4.490 & 1.940 \\
\hline 2452094.853 & 2.450 & 1.550 \\
\hline 2452096.894 & 4.560 & 1.850 \\
\hline 2452097.982 & 5.180 & 1.610 \\
\hline 2452127.919 & 1.940 & 2.200 \\
\hline 2452133.737 & 2.830 & 2.010 \\
\hline 2452160.872 & -6.120 & 2.170 \\
\hline 2452161.821 & -6.840 & 2.370 \\
\hline 2452162.787 & -8.300 & 2.370 \\
\hline 2452445.988 & 17.880 & 2.100 \\
\hline 2452537.743 & -4.900 & 2.340 \\
\hline 2452713.113 & 8.860 & 1.590 \\
\hline 2452806.027 & -4.580 & 1.990 \\
\hline 2452850.901 & 6.090 & 2.040 \\
\hline 2453179.985 & 12.360 & 1.730 \\
\hline 2453479.066 & 8.000 & 1.210 \\
\hline 2453549.857 & -2.830 & 0.880 \\
\hline 2453604.881 & -2.530 & 1.220 \\
\hline 2453838.109 & 3.550 & 1.220 \\
\hline 2453932.905 & 3.890 & 1.380 \\
\hline 2453960.873 & 3.880 & 1.630 \\
\hline 2453961.820 & -0.510 & 1.800 \\
\hline 2453981.789 & -14.390 & 1.190 \\
\hline 2453982.904 & -1.880 & 1.900 \\
\hline 2453983.831 & -8.010 & 1.220 \\
\hline 2453984.887 & -0.360 & 1.290 \\
\hline 2454248.023 & 7.800 & 2.090 \\
\hline 2454248.990 & 8.410 & 2.030 \\
\hline 2454249.945 & -3.960 & 1.730 \\
\hline 2454252.032 & -12.260 & 1.870 \\
\hline 2454255.924 & -12.790 & 1.280 \\
\hline 2454277.851 & 8.200 & 1.500 \\
\hline 2454278.896 & 11.300 & 1.700 \\
\hline 2454279.933 & 11.850 & 1.580 \\
\hline 2454294.903 & -10.850 & 1.260 \\
\hline 2454304.888 & -4.110 & 1.570 \\
\hline 2454306.037 & 2.450 & 1.600 \\
\hline 2454307.015 & -2.050 & 1.500 \\
\hline 2454308.072 & 5.540 & 1.600 \\
\hline 2454309.050 & 7.370 & 1.810 \\
\hline 2454310.042 & 8.730 & 1.720 \\
\hline 2454311.024 & 1.600 & 1.480 \\
\hline 2454312.017 & 0.860 & 1.590 \\
\hline 2454312.875 & 2.350 & 1.450 \\
\hline 2454313.875 & -1.170 & 1.320 \\
\hline 2454314.908 & 6.220 & 1.630 \\
\hline 2454318.921 & 5.360 & 1.560 \\
\hline 2454335.826 & -13.560 & 1.940 \\
\hline 2454338.888 & -16.490 & 1.790 \\
\hline 2454339.883 & -9.590 & 1.780 \\
\hline 2454343.791 & -8.170 & 1.950 \\
\hline 2454396.717 & 3.080 & 2.390 \\
\hline 2454397.729 & -4.380 & 2.130 \\
\hline 2454548.052 & 12.600 & 1.330 \\
\hline 2454549.092 & 3.010 & 1.280 \\
\hline 2454633.963 & -6.780 & 1.570 \\
\hline 2454634.903 & -5.330 & 1.650 \\
\hline 2454635.946 & -2.500 & 1.790 \\
\hline 2454636.901 & 2.170 & 1.650 \\
\hline 2454637.939 & -5.830 & 1.610 \\
\hline 2454638.838 & -5.010 & 1.450 \\
\hline 2454639.998 & 3.970 & 1.850 \\
\hline 2454641.954 & 2.760 & 1.540 \\
\hline
\end{tabular}


Table 4

(Continued)

\begin{tabular}{|c|c|c|}
\hline JD & $\begin{array}{c}\mathrm{RV} \\
\left(\mathrm{m} \mathrm{s}^{-1}\right)\end{array}$ & $\begin{array}{l}\text { Uncertainty } \\
\left(\mathrm{m} \mathrm{s}^{-1}\right)\end{array}$ \\
\hline 2454674.860 & -0.820 & 1.440 \\
\hline 2454688.892 & -4.450 & 1.710 \\
\hline 2454689.917 & 6.340 & 1.890 \\
\hline 2454717.853 & 4.220 & 2.000 \\
\hline 2454718.914 & -2.870 & 2.070 \\
\hline 2454719.866 & 1.210 & 2.040 \\
\hline 2454720.871 & 0.570 & 2.140 \\
\hline 2454721.879 & 4.620 & 2.040 \\
\hline 2454722.797 & 5.810 & 2.210 \\
\hline 2454723.814 & 2.610 & 2.120 \\
\hline 2454724.848 & 1.350 & 1.990 \\
\hline 2454968.017 & 5.210 & 0.970 \\
\hline 2455016.035 & 0.730 & 1.370 \\
\hline 2455022.084 & 1.680 & 1.670 \\
\hline 2455023.044 & 6.360 & 1.070 \\
\hline 2455024.823 & -11.730 & 0.920 \\
\hline 2455049.825 & 0.220 & 1.090 \\
\hline 2455050.858 & 3.990 & 1.650 \\
\hline 2455051.772 & 6.360 & 1.790 \\
\hline 2455052.780 & 8.540 & 1.930 \\
\hline 2455053.925 & -12.540 & 1.510 \\
\hline 2455143.692 & -3.080 & 2.620 \\
\hline 2455167.704 & 3.210 & 1.710 \\
\hline 2455259.119 & 3.760 & 1.610 \\
\hline 2455260.144 & 0.000 & 1.470 \\
\hline 2455371.991 & -5.030 & 1.070 \\
\hline 2455407.955 & -1.480 & 1.060 \\
\hline 2455463.718 & -8.590 & 1.520 \\
\hline 2455516.696 & -1.120 & 1.740 \\
\hline 2455518.722 & -3.780 & 2.320 \\
\hline 2455609.135 & 11.250 & 1.310 \\
\hline 2455638.121 & -3.930 & 0.910 \\
\hline 2455639.143 & -2.250 & 0.910 \\
\hline 2455665.046 & -5.130 & 1.190 \\
\hline 2455670.082 & -8.090 & 1.290 \\
\hline 2455721.057 & 6.840 & 1.850 \\
\hline 2455825.832 & 3.030 & 2.280 \\
\hline 2455840.748 & 3.820 & 1.390 \\
\hline 2456027.098 & 11.390 & 1.940 \\
\hline 2456116.938 & 16.010 & 1.130 \\
\hline 2456117.906 & 14.270 & 1.050 \\
\hline 2456329.167 & 5.420 & 1.970 \\
\hline 2456432.892 & -0.700 & 1.300 \\
\hline 2456433.935 & 1.610 & 1.340 \\
\hline 2456548.855 & -2.340 & 1.490 \\
\hline 2456549.793 & -7.780 & 1.430 \\
\hline 2456550.849 & -3.840 & 2.150 \\
\hline 2456551.724 & -2.520 & 1.570 \\
\hline
\end{tabular}

Table 5

Hobby-Eberly Telescope Radial Velocities for GJ 687

\begin{tabular}{lcc}
\hline \hline JD & $\begin{array}{c}\mathrm{RV} \\
\left(\mathrm{m} \mathrm{s}^{-1}\right)\end{array}$ & $\begin{array}{c}\text { Uncertainty } \\
\left(\mathrm{m} \mathrm{s}^{-1}\right)\end{array}$ \\
\hline 2452394.962 & -2.010 & 2.220 \\
2452395.925 & -3.600 & 2.600 \\
2452396.936 & -1.400 & 2.870 \\
2452400.920 & 3.800 & 2.880 \\
2452403.869 & 1.370 & 2.290 \\
\hline
\end{tabular}

Table 6

APF Radial Velocities for GJ 687

\begin{tabular}{lrc}
\hline \hline JD & $\begin{array}{c}\text { RV } \\
\left(\mathrm{m} \mathrm{s}^{-1}\right)\end{array}$ & $\begin{array}{r}\text { Uncertainty } \\
\left(\mathrm{m} \mathrm{s}^{-1}\right)\end{array}$ \\
\hline 2456484.738 & 11.650 & 0.910 \\
2456486.839 & 9.500 & 0.950 \\
2456488.845 & 11.640 & 1.120 \\
2456490.726 & 8.300 & 1.260 \\
2456492.816 & 7.020 & 0.900 \\
2456494.776 & 5.260 & 0.750 \\
2456508.764 & -8.650 & 0.780 \\
2456510.800 & -9.020 & 0.800 \\
2456513.772 & -8.920 & 0.860 \\
2456521.823 & -2.120 & 0.730 \\
2456522.790 & -2.920 & 0.930 \\
2456533.797 & 2.910 & 0.820 \\
2456551.743 & -8.410 & 0.810 \\
2456552.717 & -6.660 & 0.970 \\
2456553.727 & -7.610 & 0.850 \\
2456554.720 & -4.640 & 0.890 \\
2456564.762 & 1.530 & 0.940 \\
2456568.692 & 0.460 & 1.140 \\
2456571.657 & 0.580 & 0.940 \\
2456622.032 & -9.750 & 1.280 \\
2456677.921 & 2.390 & 0.980 \\
2456681.011 & 4.660 & 1.360 \\
\hline
\end{tabular}

\section{REFERENCES}

Anglada-Escudé, G., Arriagada, P., Vogt, S. S., et al. 2012, ApJL, 751, L16 Balan, S. T., \& Lahav, O. 2009, MNRAS, 394, 1936

Baluev, R. V. 2012, MNRAS, 422, 2372

Baraffe, I., Chabrier, G., Allard, F., \& Hauschildt, P. H. 1998, A\&A, 337, 403

Batalha, N. M., Rowe, J. F., Bryson, S. T., et al. 2013, ApJS, 204, 24

Boisse, I., Bonfils, X., \& Santos, N. C. 2012, A\&A, 545, A109

Boyajian, T. S., von Braun, K., van Belle, G., et al. 2012, ApJ, 757, 112

Butler, R. P., Marcy, G. W., Williams, E., et al. 1996, PASP, 108, 500

Butler, R. P., Vogt, S. S., Marcy, G. W., et al. 2004, ApJ, 617, 580

Charbonneau, P. 2010, LRSP, 7, 3

Cutri, R. M., Skrutskie, M. F., van Dyk, S., et al. 2003, 2MASS All Sky Catalog of Point Sources (Pasadena, CA: NASA/IPAC Infrared Science Archive)

Dressing, C. D., \& Charbonneau, D. 2013, ApJ, 767, 95

Dumusque, X., Pepe, F., Lovis, C., et al. 2012, Natur, 491, 207

Eaton, J. A., Henry, G. W., \& Fekel, F. C. 2003, in The Future of Small Telescopes In The New Millennium, ed. T. D. Oswalt (Astrophysics and Space Science Library, Vol. 288; Dordrecht: Kluwer), 189

Efron, B. 1979, AnSta, 7, 1

Endl, M., Cochran, W. D., Tull, R. G., \& MacQueen, P. J. 2003, AJ, 126, 3099 Fischer, D. A., \& Valenti, J. 2005, ApJ, 622, 1102

Ford, E. B. 2005, AJ, 129, 1706

Ford, E. B., \& Gaudi, B. S. 2006, ApJL, 652, L137

Gonzalez, G. 1997, MNRAS, 285, 403

Gregory, P. C. 2011, MNRAS, 415, 2523

Henry, G. W. 1999, PASP, 111, 845

Henry, G. W., Fekel, F. C., \& Hall, D. S. 1995, AJ, 110, 2926

Henry, G. W., Fekel, F. C., Kaye, A. B., \& Kaul, A. 2001, AJ, 122, 3383

Henry, T. J., \& McCarthy Jr., D. W. 1993, AJ, 106, 773

Jenkins, J. S., Jones, H. R. A., Tuomi, M., et al. 2013, ApJ, 766, 67

Jenkins, J. S., Ramsey, L. W., Jones, H. R. A., et al. 2009, ApJ, 704, 975

Johnson, J. A., \& Apps, K. 2009, ApJ, 699, 933

Kukla, A. 2010, Extraterrestrials: A Philosophical Perspective (Lanham, MD: Lexington Books)

Lissauer, J. J., Ragozzine, D., Fabrycky, D. C., et al. 2011, ApJS, 197, 8

Mayor, M., Marmier, M., Lovis, C., et al. 2011, arXiv:1109.2497

Mayor, M., Udry, S., Lovis, C., et al. 2009, A\&A, 493, 639

Meschiari, S., Wolf, A. S., Rivera, E., et al. 2009, PASP, 121, 1016

Meschiari, S., Wolf, A. S., Rivera, E., et al. 2012, ascl soft, 1210.018

Neves, V., Bonfils, X., Santos, N. C., et al. 2013, A\&A, 551, A36

Paulson, D. B., Saar, S. H., Cochran, W. D., \& Henry, G. W. 2004, AJ, 127,1644 
Petigura, E. A., Howard, A. W., \& Marcy, G. W. 2013, PNAS, 110, 19273

Press, W. H., Teukolsky, S. A., Vetterling, W. T., \& Flannery, B. P. 1992, Numerical Recipes in C: The Art of Scientific Computing (2nd ed.; Cambridge: Cambridge Univ. Press)

Queloz, D., Henry, G. W., Sivan, J. P., et al. 2001, A\&A, 379, 279

Rivera, E. J., Laughlin, G., Butler, R. P., et al. 2010, ApJ, 719, 890

Roberts, D. H., Lehar, J., \& Dreher, J. W. 1987, AJ, 93, 968

Rojas-Ayala, B., Covey, K. R., Muirhead, P. S., \& Lloyd, J. P. 2012, ApJ, 748,93

Scargle, J. D. 1982, ApJ, 263, 835

Schlaufman, K. C., \& Laughlin, G. 2010, A\&A, 519, A105
Sousa, S. G., Santos, N. C., Israelian, G., Mayor, M., \& Udry, S. 2011, A\&A, 533, A141

Triaud, A. H. M. J., Anderson, D. R., Collier Cameron, A., et al. 2013, A\&A, 551, A80

Vogt, S. S., Allen, S. L., Bigelow, B. C., et al. 1994, Proc. SPIE, 2198, 362

Vogt, S. S., Butler, R. P., Rivera, E. J., et al. 2014a, ApJ, 787, 97

Vogt, S. S., Radovan, M., Kibrick, R., et al. 2014b, ApJ, 787, 97

Wright, J. T. 2005, PASP, 117, 657

Wright, J. T., Fakhouri, O., Marcy, G. W., et al. 2011, PASP, 123, 412

Wright, J. T., Marcy, G. W., Butler, R. P., \& Vogt, S. S. 2004, ApJS, 152,261 